\title{
A Numerical Framework for Isotropic and Anisotropic Flexible Flapping Wing Aerodynamics and Aeroelasticity
}

\author{
Hikaru Aono ${ }^{*}$, Chang-kwon Kang ${ }^{\dagger}$, Carlos E. S. Cesnik ${ }^{\ddagger}$, and Wei Shyy ${ }^{\S}$ \\ Department of Aerospace Engineering, University of Michigan, Ann Arbor, MI, 48105
}

\begin{abstract}
A numerical framework is presented to simulate rigid and flexible flapping wings. The fluid-structure interaction is realized by coupling a Navier-Stokes solver to a geometrically nonlinear co-rotational structural solver. In the coupled fluid-structure interaction interface the aerodynamic loading and deformed wing surface are shared by two solvers within each time step. A remeshing method based on radial basis function is implemented to handle large deformations, preserve grid quality, and execute economically. Flexible flapping wing operating in both vacuum and air is considered to shed light on inertial and aerodynamic loading-induced shape deformation. Impacts of flapping isotropic wing with single degree of freedom rotation on aerodynamic force generation are presented for $R e=1.5 \times 10^{3}$ and $k=$ 0.56. The results indicate: 1) the flexibility-induced pitching angle promotes thrust generation, and 2) the increase of wing velocity due to large bending motion enhances aerodynamic force by increasing pressure differences. For a wing of anisotropic mechanical properties, highly three-dimensional wing deformation is observed when the wing accelerates or decelerates in absent of aerodynamic loadings.
\end{abstract}

\section{Nomenclature}

\begin{tabular}{|c|c|c|}
\hline$A R$ & $=$ aspect ratio, $b^{2} / S$ & [1] \\
\hline$A_{i j}^{e}$ & $=$ element extensional stiffness matrix & {$[\mathrm{Pa} \mathrm{m}]$} \\
\hline$b_{m}$ & $=$ mean wing span & {$[\mathrm{m}]$} \\
\hline$B_{i j}^{e}$ & $=$ membrane-bending coupling stiffness matrix & {$\left[\mathrm{Pa} \mathrm{m}^{2}\right]$} \\
\hline$C_{L}$ & $=$ lift coefficient, $F_{1} /\left(1 / 2 \rho_{f} U_{\mathrm{ref}}^{2} S\right)$ & {$[1]$} \\
\hline$c_{m}$ & $=$ mean chord length & {$[\mathrm{m}]$} \\
\hline$C_{T}$ & $=$ thrust coefficient, $-F_{2} /\left(1 / 2 \rho_{f} U_{\text {ref }}^{2} S\right)$ & [1] \\
\hline$D_{i j}^{e}$ & $=$ bending stiffness matrix & {$\left[\mathrm{Pa} \mathrm{m}^{3}\right]$} \\
\hline$E$ & $=$ Young's modulus of an isotropic material & {$[\mathrm{Pa}]$} \\
\hline$E_{11}$ & $=$ Young's modulus of an orthotropic material in the $x_{1}$ direction & {$[\mathrm{Pa}]$} \\
\hline$E_{22}$ & $=$ Young's modulus of an orthotropic material in the $x_{2}$ direction & {$[\mathrm{Pa}]$} \\
\hline$f$ & $=$ motion frequency & {$[1 / \mathrm{s}]$} \\
\hline$f_{p}$ & $=$ distributed external force & {$[\mathrm{N}]$} \\
\hline$G_{12}$ & $=$ Shear modulus of an orthotropic material in the $x_{1} x_{2}$ plane & {$[\mathrm{Pa}]$} \\
\hline$h_{s}$ & $=$ thickness of a plate & {$[\mathrm{m}]$} \\
\hline$k$ & $=$ reduced frequency, $\pi f c_{m} / U_{\text {ref }}$ & [1] \\
\hline$N$ & $=$ mesh refinement factor & [1] \\
\hline$N_{b}$ & $=$ number of boundary nodes with specified displacements & [1] \\
\hline$N_{\text {CSD }}$ & $=$ number of nodes on structural mesh & [1] \\
\hline$N_{v}$ & $=$ number of internal nodes & [1] \\
\hline
\end{tabular}

\footnotetext{
* Post- doctoral Research Fellow, Department of Aerospace Engineering, University of Michigan, AIAA Member

${ }^{\dagger}$ Graduate Student Research Assistant, Department of Aerospace Engineering, University of Michigan, AIAA Student Member

¥Professor, Department of Aerospace Engineering, University of Michigan, AIAA Associate Fellow

${ }^{\S}$ Clarence L. "Kelly" Johnson Collegiate Professor and Chair, Department of Aerospace Engineering, University of Michigan, AIAA Fellow.
} 


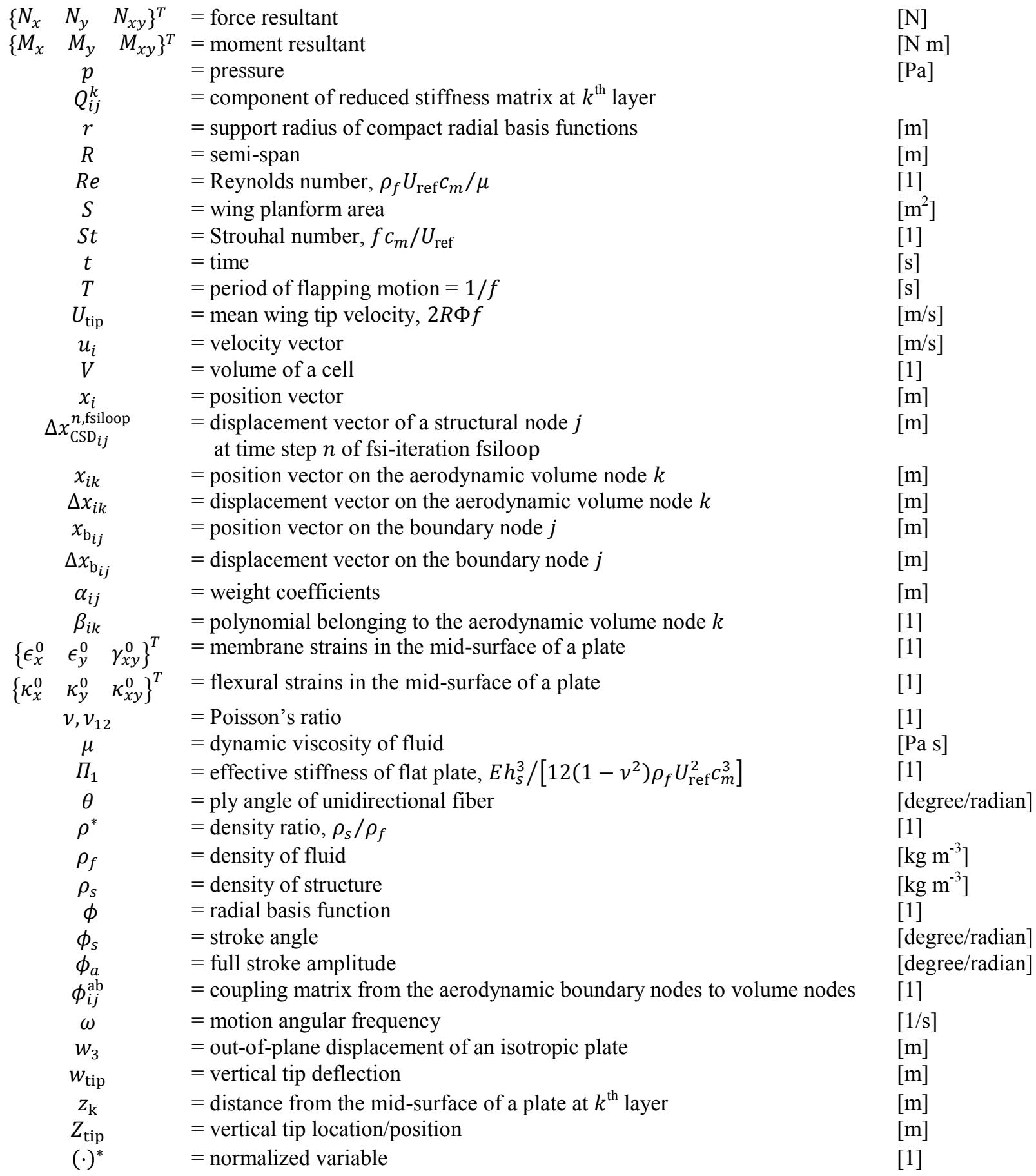

\section{Introduction}

ICRO air vehicles (MAVs) are flyers with maximum dimensions of $1.5 \times 10^{-1} \mathrm{~m}$ that can lead to revolutionary improvements in remote sensing and information gathering capabilities both in military as well in civilian applications. As the size of the flyer is orders of magnitudes smaller than a conventional aircraft, the fluid dynamics, hence the lift generation, and the flight control governing a MAV change considerably ${ }^{1,2}$ : Smaller sizes and lower flight speeds lead to lower Reynolds numbers, and higher sensitivity to wind gust effects. Moreover, wings are in general flexible and deform during flight. Consequently, design of a high performance and robust MAV become a highly nonlinear complex process that requires solid understanding of aerodynamics, structural dynamics, control, and interactions of these. 
In the nature, biological flyers, such as insects and birds, share similar dimensions and flight characteristics as MAVs ${ }^{1}$. The physics exhibited by these biological flyers thus have potential to be applied in MAV design ${ }^{1}$. Unsteady aerodynamic mechanisms such as the generation of a leading edge vortex (LEV), wing-wake interaction, and three-dimensional flow features, such as tip vortex-vortex interactions, all contribute significantly to affect the aerodynamic force generation ${ }^{2}$. Another remarkable mechanism that the biological flyers seem to be using is the wing flexibility. Studies have been performed to shed light on the interplay between the structural flexibility and the resulting aerodynamic forces ${ }^{3-6}$. For example, Combes and Daniel ${ }^{7,8}$ have shown that a variety of insects exhibit anisotropy in their wing structures based on static response tests. The spanwise flexural rigidity was 1 or 2 orders of magnitudes larger than the chordwise flexural rigidity. Recent experimental and numerical ${ }^{4}$ show that the chordwise flexibility affects the redistribution of the resulting aerodynamic forces in the lift and the thrust directions. For example, if the airfoil shape undergoes deformation then the camber of the airfoil may change, leading to an effective geometry of the airfoil, and combined with the pitching angle the direction of the net force can be adjusted in favor of the thrust generation. Furthermore, for a range of spanwise flexibility, deformed airfoil shapes along the spanwise direction and the correct direction of the motion are seen to enhance the thrust of a plunging wing ${ }^{9,10}$. The fluid-structure density ratio affects the thrust generation: $\mathrm{Zhu}^{11}$ showed numerically that the thrust and the propulsive efficiencies increased for a plunging chordwise flexible airfoil in water than immersed in air. Hence it is seen that the flexibility, including the density ratio can be utilized to control resulting aerodynamic forces. However the precise underlying physics of aeroelastic coupling for flapping wings and its applicability to the MAV designs are yet to be understood. Shyy et $a l^{2}$ offer an extensive review on the aerodynamics of rigid and flexible wings.

Recently, systematic methodologies of experiment have been developed to optimize thrust generated by single degree-of-freedom (DOF) flapping wing via variation of wing structures ${ }^{12}$. In these studies, several combinations of flapping wing frequency, amplitude, and wing structure are examined. The conclusion made from the experimental studies is that for flapping wing structure optimization in hovering flight on size scale of humming birds or insects, each wing structure must be tailored according to a certain kinematics (with a desired flapping frequency and amplitude) and optimized for performance values such as either lift by drag or flight efficiency. Scaling parameters resulting from dimensional analysis and by non-dimensionalizing the governing equations by relevant physical variables, often give qualitative characteristics of the model as well as the requirement for dynamic similarity of the flow ${ }^{13}$. Depending on the type of the model and the governing equations the resulting set of scaling parameters may vary. Shyy et al. ${ }^{1,2}$ have considered the Navier-Stokes equations with out-of-plane motion of an isotropic plate, Ishihara et al. ${ }^{5,14}$, the Navier-Stokes equations and the wing structure as a linear isotropic elastic body to study the flexibility effect on wing pitch changes in dipteran flapping flight, and Lentink and Dickinson ${ }^{15}$ transformed the Navier-Stokes equation in the wing-fitted frame of reference and focused on the rotational motion of the wing. The scaling parameters related to the unsteady aerodynamics, such as the Reynolds number, the Strouhal number, or the reduced frequency have been explored previously ${ }^{1,2}$, still the parameter-space involving the scaling parameters for the fluid-structure interaction needs to be mapped out in a systematic fashion to understand the role of flexibility on flapping wing force generation.

Different levels of fidelity and diverse numerical algorithms, depending on the objective of the study and accuracy and cost of the computations, can be incorporated into a numerical framework of flexible flapping wing simulations. High-fidelity framework can be used to evince the rich and complex physics behind flexible flapping wing aerodynamics, while a low-fidelity model can be used for quick yet reliable design optimization of a complex and multi-dimensional design space. The aeroelastic coupling can be based on a time-domain partitioned process where the interaction between the fluid and the solid fields occurs at a shared boundary iteratively at a given time step. An advantage is that for the solutions of both fields, which are described by different nonlinear partial differential equations, well-established solvers can be used. Tang et al. ${ }^{16}$, Chimakurti et al. ${ }^{10,17}$, and Aono et al. ${ }^{4,18}$ have coupled an in-house finite-volume based Navier-Stokes solver to a beam model ${ }^{16}$, a commercial nonlinear finite-element solver, MSC.Marc ${ }^{10}$, a geometrically nonlinear active beam solver ${ }^{10}$, and a co-rotational shell finite element model ${ }^{17,4,18}$, respectively. Furthermore, Gordnier et al. ${ }^{19}$ coupled an in-house high-order Navier-Stokes solver to a geometrically nonlinear active beam solver, McClung et al. ${ }^{20}$ developed the OVERFLOW Navier-Stokes solver to a modal representation of two-dimensional beam, and Stanford et $a .^{21}$ combined a two-dimensional quasisteady blade element model to a nonlinear co-rotational beam model.

Some of the numerical challenges associated with aeroelastic coupling of highly deformable three-dimensional flapping wings are that large number of cells are required to resolve the necessary flow field phenomena such as vorticity generation near the wing as well as convection and diffusion of large scale vortical structures away from the wing and that large flapping amplitudes and deformations lead to large mesh deformations that require numerically robust and efficient remeshing algorithms. To obtain the three-dimensional flow solutions with the number of cells in the orders of $\mathrm{O}\left(10^{6}\right)$ a Navier-Stokes equations solver is often parallelized, which introduces 
obvious implementation challenges for the problems involving moving meshes: how to efficiently communicate information between multiple processors about the displacements stored in other processors to remesh the grid; and how to provide interface for the communication for the fluid-structure interaction involving two distinct solvers, where one or both solvers are parallelized. Two strategies have been studied for unstructured grids. In the first category the computational mesh is considered as an elastic solid, where the new nodal displacements are obtained by solving the structural dynamics equations. For example, Stein and Tezduyar ${ }^{22}$ proposed to solve the linear elastostatics equations with a stiffening factor to reduce the volumetric distortion to maintain the mesh quality in finite element formulation. Smith and $\mathrm{Wright}^{23}$ recast the linear elastostatics equations in finite-volume discretization, which involves similar terms as the Navier-Stokes equations, so that the remeshing algorithm could be implemented based on the readily available momentum equation solver as a template. In the second category the nodal displacements are interpolated using Radial Basis Functions (RBF) based on the displacements given at the boundary conditions, either prescribed or from the structural dynamics solver. This method, as the cell connectivity information is not required, can be applied to structured and unstructured meshes and is seen to keep initial mesh qualities well and efficiently ${ }^{24-27}$.

Chimakurthi et al. ${ }^{10}$ have presented a computational framework capable of computing flexible flapping wing aerodynamics and aeroelasticity. They have detailed fluid and structural solvers as well as the coupling strategies. Aono et al. ${ }^{4}$ have further developed the techniques and investigated the dynamic characteristics associated with spanwise flexibility of a plunging wing. Chordwise flexibility of a plunging wing is discussed in Shyy et al. ${ }^{2}$. The computational framework is restricted to isotropic wing structures. In the present paper, we have advanced the computational capabilities presented in Ref 10. Specifically, we have incorporated an unstructured grid NavierStokes solver and an anisotropic structural solver. The interface between fluid and structure solvers is based on that reported in Ref 10. Furthermore, new remeshing techniques, similar to that reported by De Boer et al. ${ }^{24}$ and Rendall

and Allen ${ }^{25-27}$, have been developed and tested. The added capabilities can handle a variety of wing structures with large flapping translation and rotation. In order to shed light on the role of inertial and aerodynamic loading-induced shape deformation associated with a flapping flexible wing, wings operating in both vacuum and air are considered. To account for the anisotropic nature of wing structures, a composite structural model is implemented based on the classical laminate plate theories ${ }^{39}$. Structural dynamic response of flapping anisotropic wing models in vacuum are presented and compared with the experimental data ${ }^{12}$. Furthermore, a remeshing method using the RBF interpolation is presented that is able to efficiently deform large number of nodes under large wing movements, while keeping the initial mesh qualities. The fluid flow obtained by using a finite-volume pressure-based Navier-Stokes solver and the structural dynamics solver uses the finite element method with an optimal membrane element and a discrete Kirchhoff triangle plate bending element in a co-rotational formulation to effectively simulate a thin flexible wing ${ }^{17}$. In the coupled fluid-structure interaction interface the aerodynamic loading and deformed wing surface is shared by two solvers within each time step. The relevance of this framework is three-fold. The computed fluid flow field and the structural response can be studied to understand the nonlinear coupled field problem for flapping wing flyers, also these results can function as a validation for lower order tools or experimental results. Moreover, this framework can serve as a tool to construct a quick and reliable design guideline for MAV applications.

\section{Methodology}

\section{A. Computational fluid dynamics}

\section{Governing equations}

The governing equations for the fluids are unsteady three-dimensional Navier-Stokes equations with constant density and viscosity shown in Eqs. (1) and (2),

$$
\begin{gathered}
\frac{\partial u_{j}}{\partial x_{j}}=0 \\
\frac{\partial u_{i}}{\partial t}+\frac{\partial u_{j} u_{i}}{\partial x_{j}}=-\frac{1}{\rho_{f}} \frac{\partial p}{\partial x_{i}}+\mu \frac{\partial}{\partial x_{j}}\left(\frac{\partial u_{i}}{\partial x_{j}}\right)
\end{gathered}
$$

where $u_{i}$ is the velocity vector, $x_{i}$ is the position vector, $t$ is the time, $p$ is the pressure, $\rho_{f}$ is the fluid velocity, and $\mu$ the viscosity of the fluid. The Eqs (1) and (2) are solved with in-house codes ${ }^{18,28,29}$, which are three-dimensional pressure-based finite-volume solvers. The parallelization is realized using the Loci framework ${ }^{30}$. It employs implicit first or second order time stepping and treats convection terms using the second order upwind-type scheme $e^{28,31,32}$, 
pressure and viscous terms using second order schemes. The system of equations resulting from the linearized momentum equations are handled with the symmetric Gauss-Seidel ${ }^{33}$ solver which has relatively low memory requirements. The pressure correction equation ${ }^{28,}{ }^{35}$ is solved with the GMRES linear solver with Jacobi preconditions provided by PETSc ${ }^{35}$. The geometric conservation law, a necessary consideration in domains with moving boundaries, is satisfied ${ }^{36,37}$. The mesh deformations are realized using either Radial Basis Function (RBF) or thin plate spline interpolations ${ }^{16-18}$.

\section{Remeshing}

De Boer et $_{\text {al. }}{ }^{24}$ and Rendall and Allen ${ }^{25-27}$ have shown the effectiveness of using RBF interpolation method to remesh the unstructured grids. There are two distinctive advantages: i) Connectivity information is unnecessary for the RBF interpolation method, that means any arbitrary order of nodal information will result in the same mesh. However, ordering the nodes based on the distances between the nodes will make the linear system more diagonally dominant for the compact $\mathrm{RBFs}^{24}$, which will reduce the computational time. ii) The resulting mesh keeps the original mesh quality, especially the orthogonality near the moving boundary surface ${ }^{24,25}$ which is important in viscous fluid computation where the cells are usually stretched and clustered to capture small length scale flow phenomena.

Given the boundary displacement vector $\Delta x_{\mathrm{b}_{i j}}$ at the node $j$, with the position $x_{\mathrm{b}_{i j}}$ either on the aerodynamic surface mesh of a flexible body, or any other prescribed boundary surface, for instance zero displacements at the tunnel wall, the displacement vector, $\Delta x_{i k}$, of a node $k$, with the position $x_{i k}$, of the aerodynamic volume mesh with respect to the initial grid, is given by Eq. (3) with summation of repeated indices as

$$
\Delta x_{i k}=\alpha_{i j} \phi_{k j}^{\mathrm{ab}}+\beta_{i k}
$$

where index $i$ represents the three components in three-dimensional space, $\phi_{i j}^{\mathrm{ab}}$ is the coupling matrix from the aerodynamic surface nodes to the aerodynamic volume nodes given as

$$
\phi_{k j}^{\mathrm{ab}}=\phi\left(\left\|x_{\mathrm{a}_{l k}}-x_{\mathrm{b}_{l j}}\right\|_{l}\right)
$$

with $\|\cdot\|$ being the Euclidean distance measure acting on the index $l=1,2,3, \phi$ is a radial basis function, and $\beta_{i k}$ is a polynomial. If the radial function is conditionally positive definite, and $\beta_{i k}$ is a linear polynomial, then a unique remeshing will result ${ }^{24,38}$. In the current study a linear polynomial will be used:

$$
\beta_{i k}=\beta_{i k}^{0}+\beta_{i}^{j} x_{\mathrm{a}_{j k}}
$$

For remeshing the compact $C^{2}$ RBF constructed by Wendland ${ }^{38}$ will be used in the current study motivated by the previous results ${ }^{24,25,38}$ that showed it to be computationally efficient while being able to retain the mesh qualities, given by Eq. (4) as

$$
\phi(x)=\left(1-\frac{x}{r}\right)^{4}\left(\frac{4 x}{r}+1\right)
$$

where $r$ is the support radius of this compact function. As the support radius increases a node acquires more information about the displacements of neighboring nodes, leading to better approximation. On the other hand if the support radius is small the resulting linear system will be sparse, hence the computational cost would decrease. However if $r$ is too small, negative jacobian or volume of a cell might occur. All RBFs, compact and global, listed in Ref. 24 are available in the current framework for interpolation purposes.

The unknowns in Eq. (3) are the weight coefficients, $\alpha_{i j}\left(i=1,2,3, j=1, \ldots, N_{\mathrm{b}}\right)$, and the polynomial coefficients, $\beta_{i}^{j}$, that can be solved by the requirements that the prescribed nodal displacements must be recovered by the RBF interpolation at these nodes, i.e.,

$$
\Delta x_{\mathrm{b}_{i k}}=\alpha_{i j} \phi\left(\left\|x_{\mathrm{b}_{l k}}-x_{\mathrm{b}_{l j}}\right\|_{l}\right)+\beta_{i k}^{0}+\beta_{i}^{j} x_{\mathrm{b}_{j k}}
$$

and the additional requirement for unique solution ${ }^{38}$, 


$$
\alpha_{i j} 1_{j}=0_{i}, \quad \alpha_{i j} x_{\mathrm{b}_{j k}}=0_{i k}
$$

where $1_{j}$ is a summation vector that sums all elements along $j, 0_{i}$ the zero vector over the index $i$, and $0_{i k}$ the zero matrix over the index $i k$. In vector notation the resulting linear system is shown in Eq. (5) and is exactly the same as found in Refs 24, 38:

$$
\left[\begin{array}{cc}
\mathbf{M}_{\mathrm{b}, \mathrm{b}} & \mathbf{Q}_{\mathrm{b}} \\
\mathbf{Q}_{\mathrm{b}}^{T} & \mathbf{0}
\end{array}\right]\left[\begin{array}{l}
\boldsymbol{\alpha} \\
\boldsymbol{\beta}
\end{array}\right]=\left[\begin{array}{c}
\Delta \boldsymbol{x}_{\mathrm{b}} \\
\mathbf{0}
\end{array}\right]
$$

with for each $i$ index

- $\phi\left(\left\|x_{\mathrm{b}_{l k}}-x_{\mathrm{b}_{l j}}\right\|_{l}\right) \rightarrow \mathbf{M}_{\mathrm{b}, \mathrm{b}}$,

- $\left[\begin{array}{ll}1_{j} & x_{\mathrm{b}_{j k}}\end{array}\right] \rightarrow \mathbf{Q}_{\mathrm{b}}$,

- $\alpha_{i j} \rightarrow \boldsymbol{\alpha}_{i}, \beta_{i}^{j} \rightarrow \boldsymbol{\beta}_{i}, \Delta x_{\mathrm{b}_{i k}} \rightarrow \Delta \boldsymbol{x}_{\mathrm{b}_{i}}$.

This linear system is solved using PETSc's GMRES iterative solver with Jacobian preconditioner using multiple processors.

To capture the three-dimensional flow phenomena the number of cells is typically in the order of millions. To enhance the computational speed the fluid-solver is parallelized using MPI where the computational domain is decomposed optimally for the computation of velocity and pressure fields. This implies that the boundary nodes on the moving surfaces are randomly distributed among the processors. To implement the RBF interpolation remeshing method efficiently in parallel the following steps are taken by noting that the nodal coordinates on the boundary surfaces, $x_{b_{i j}}$, stay stationary in time:

1. Preprocessing step:

a. $\quad x_{b_{i j}} \rightarrow \mathbf{Q}_{\mathrm{b}}$ : identify the local nodes on the boundary surfaces. Using ALLGATHER operation assemble and distribute the coordinates of all nodes on the boundary surfaces into a matrix $\mathbf{Q}_{\mathrm{b}}$.

b. $\quad\left(x_{b_{i j}}, \mathbf{Q}_{\mathrm{b}}\right) \rightarrow \phi\left(\left\|x_{\mathrm{b}_{l k}}-x_{\mathrm{b}_{l j}}\right\|_{l}\right)=\mathbf{M}_{\mathrm{b}, \mathrm{b}}$ : iterate over the local nodes on the boundary surface and by computing the distances to all elements in $\mathbf{Q}_{\mathrm{b}}$ construct the local partition of the system matrix having a sparse matrix structure provided by PETSc.

\section{Remeshing:}

a. $\quad \Delta \boldsymbol{x}_{\mathrm{b}} \rightarrow\left(\boldsymbol{\alpha}_{i}, \boldsymbol{\beta}_{i}\right)$ : solve the linear system to obtain the weight and the polynomial coefficients locally. Using the ALLGATHER operation, distribute the coefficients globally.

b. $\left(\boldsymbol{\alpha}_{i}, \boldsymbol{\beta}_{i}, \mathbf{Q}_{\mathrm{b}}, x_{b_{i j}}\right) \rightarrow \Delta x_{i k}$ : Compute the nodal displacements for all volume nodes by applying Eq. (3).

Since the assembly of the system matrix is performed before the time integration begins, and the parallel communication during the computation is only needed for the linear solver and for the ALLGATHER operation of the coefficients, $\boldsymbol{\alpha}_{i}$ and $\boldsymbol{\beta}_{i}$, this method is suitable for parallelization. Further improvements can be obtained by using the Schur-complement techniques which involve the inversion of $\mathbf{M}_{\mathrm{b}, \mathrm{b}}$ has the potential to accelerate the remeshing process since $\mathbf{M}_{\mathrm{b}, \mathrm{b}}$ is a positive-definite symmetric matrix so that well-known iterative solvers can be used such as PCG, and during the time integration only matrix multiplications are needed to obtain the new displacements for the volume nodes ${ }^{25}$.

\section{B. Structural dynamics}

The nonlinear structural dynamics are solved using a flexible multi-body type finite element analysis of a flapping wing using triangular facet shell elements. The rigid-body motions are prescribed in the global frame of reference in addition to a co-rotational framework to account for the geometric nonlinearities. By applying the corotational frame transformations the motion of an element is decomposed into the rigid-body motion part and the pure deformation part. By using the linear elasticity theory for the latter part, the co-rotational formulation can efficiently solve for the structural dynamics with small strains, yet large rotations. A linear combination of an optimal membrane element and a discrete Kirchhoff triangle plate bending element is employed for the elastic 
stiffness of a shell element. Full details of this algorithm can be found in Ref. 17. For example, the governing equations related to out-of-plane displacement of an isotropic flat plate may be given by Eq (6),

$$
\rho_{s} h_{s} \frac{\partial^{2} w_{3}}{\partial t^{2}}-\frac{E h_{s}^{3}}{12\left(1-v^{2}\right)}\left(\frac{\partial^{4} w_{3}}{\partial x_{1}^{4}}+2 \frac{\partial^{4} w_{3}}{\partial x_{1}^{2} \partial x_{2}^{2}}+\frac{\partial^{4} w_{3}}{\partial x_{2}^{4}}\right)=-f_{p}
$$

where $w_{3}$ is the displacement in the $x_{3}$ direction, $\rho_{s}$ is the density of the flat plate, $h_{s}$ is the thickness of the plate, $E$ is the Young's modulus of the material, $v$ is the Poisson's ratio of the plate, and $f_{p}$ the distributed transverse load on the plate.

To extend the isotropic structural model to an anisotropic structural model, extension stiffness, membranebending coupling stiffness, and bending stiffness are constructed based on the classical laminate theories ${ }^{39}$ and the global stiffness matrix is assembled from both isotropic and orthotropic materials. A layer of unidirectional material is considered with directions $\left(\begin{array}{llll}x^{\prime} & y^{\prime} & z^{\prime}\end{array}\right)$, the plane $\left(\begin{array}{ll}x^{\prime} & y^{\prime}\end{array}\right)$ being identified with the plane of the layer and the direction $x^{\prime}$ with the direction of fibers as shown in Figure 1. In order to characterize the elastic properties of a layer, expressing them in the reference axes $\left(\begin{array}{llll}x & y & z\end{array}\right)$ of the laminate, the direction of the fibers making an angle $\theta$ with the direction $x$. This reference system is usually referred to as the system $\left(\begin{array}{lll}x & y & z\end{array}\right)$. The relationship between the off-axis reduced stiffness constants and those written in terms of the principal axes only bring in 1, 2, and 6 components of the stresses and strains. The reduced stiffness constants of a unidirectional or orthotropic composite, off its principal directions may be written as:

$$
\begin{aligned}
& Q_{11}^{\prime k}=Q_{11}^{k} \cos ^{4} \theta^{k}+Q_{22}^{k} \sin ^{4} \theta^{k}+2\left(Q_{12}^{k}+2 Q_{66}^{k}\right) \sin ^{2} \theta^{k} \cos ^{2} \theta^{k} \\
& Q_{12}^{\prime k}=\left(Q_{11}^{k}+Q_{22}^{k}-4 Q_{66}^{k}\right) \sin ^{2} \theta^{k} \cos ^{2} \theta^{k}+Q_{12}^{k}\left(\cos ^{4} \theta^{k}+\sin ^{4} \theta^{k}\right) \\
& Q_{16}^{\prime k}=\left(Q_{11}^{k}-Q_{12}^{k}-2 Q_{66}^{k}\right) \sin \theta^{k} \cos ^{3} \theta^{k}+\left(Q_{12}^{k}-Q_{22}^{k}+2 Q_{66}^{k}\right) \sin ^{3} \theta^{k} \cos \theta^{k} \\
& Q_{22}^{\prime k}=Q_{11}^{k} \sin ^{4} \theta^{k}+Q_{22}^{k} \cos ^{4} \theta^{k}+2\left(Q_{12}^{k}+2 Q_{66}^{k}\right) \sin ^{2} \theta^{k} \cos ^{2} \theta^{k} \\
& Q_{26}^{\prime k}=\left(Q_{11}^{k}-Q_{12}^{k}-2 Q_{66}^{k}\right) \cos \theta^{k} \sin ^{3} \theta^{k}+\left(Q_{12}^{k}-Q_{22}^{k}+2 Q_{66}^{k}\right) \cos ^{3} \theta^{k} \sin \theta^{k} \\
& Q_{66}^{\prime k}=\left[Q_{11}^{k}+Q_{22}^{k}-2\left(Q_{12}^{k}+Q_{66}^{k}\right)\right] \sin ^{2} \theta^{k} \cos ^{2} \theta^{k}+Q_{66}^{k}\left(\sin ^{4} \theta^{k}+\cos ^{4} \theta^{k}\right)
\end{aligned}
$$

where $Q_{11}^{k}=E_{1}^{k} /\left(1-\frac{E_{2}^{k}}{E_{1}^{k}}\left(v_{12}^{k}\right)^{2}\right), Q_{22}^{k}=E_{2}^{k} /\left(1-\frac{E_{2}^{k}}{E_{1}^{k}}\left(v_{12}^{k}\right)^{2}\right), Q_{12}^{k}=v_{12}^{k} Q_{22}^{k}$, and $Q_{66}^{k}=G_{12}^{k}$,respectively. The force-strain relationship for a thin laminate plate based the classical laminate theory ${ }^{39}$ is

$$
\left\{\begin{array}{c}
N_{x} \\
N_{y} \\
N_{x y} \\
M_{x} \\
M_{y} \\
M_{x y}
\end{array}\right\}=\left[\begin{array}{ll}
A^{e} & B^{e} \\
B^{e} & D^{e}
\end{array}\right]\left\{\begin{array}{c}
\epsilon_{x}^{0} \\
\epsilon_{y}^{0} \\
\gamma_{x y}^{0} \\
\kappa_{x}^{0} \\
\kappa_{y}^{0} \\
\kappa_{x y}^{0}
\end{array}\right\}
$$

where $\left\{\begin{array}{lll}\epsilon_{x}^{0} & \epsilon_{y}^{0} & \gamma_{x y}^{0}\end{array}\right\}^{T}$ and $\left\{\begin{array}{lll}\kappa_{x}^{0} & \kappa_{y}^{0} & \kappa_{x y}^{0}\end{array}\right\}^{T}$ are the membrane and flexural strains in the mid-surface of the plate. $\left\{\begin{array}{lll}N_{x} & N_{y} & N_{x y}\end{array}\right\}^{T}$ and $\left\{\begin{array}{lll}M_{x} & M_{y} & M_{x y}\end{array}\right\}^{T}$ are the force resultants and moment resultants, respectively. $A^{e}, B^{e}$ and $D^{e}$ are the extensional stiffness matrix, membrane-bending coupling stiffness matrix, and bending stiffness matrix, respectively ${ }^{39}$ :

$$
\begin{aligned}
A_{i j}^{e} & =\sum_{k=1}^{n}\left(z_{k}-z_{k-1}\right) Q_{i j}^{\prime k} \\
B_{i j}^{e} & =\sum_{k=1}^{n} \frac{1}{2}\left(z_{k}^{2}-z_{k-1}^{2}\right) Q_{i j}^{\prime k} \\
D_{i j}^{e} & =\sum_{k=1}^{n} \frac{1}{3}\left(z_{k}^{3}-z_{k-1}^{3}\right) Q_{i j}^{\prime k}
\end{aligned}
$$


where $z_{k}$ is measured from the mid-plane surface. It should be noted that in current study membrane-bending coupling matrix is set to zero because symmetric laminates are considered. Then, the stiffness matrix for the composite shell element is obtained as a superposition of the stiffnesses corresponding to that of the optimal membrane element (OPT) and a discrete Kirchoff triangle (DKT) plate-bending element.

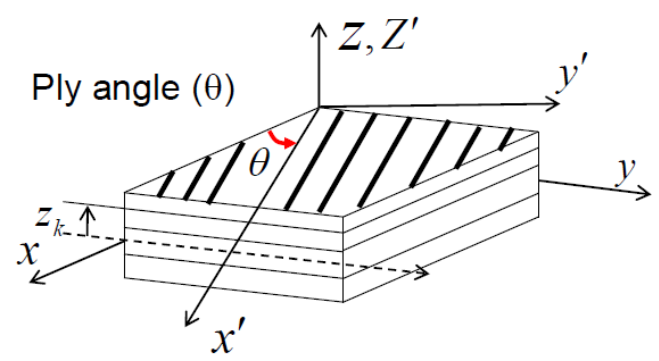

Figure 1 The principal directions $\left(\begin{array}{lll}x^{\prime} & y^{\prime} & z^{\prime}\end{array}\right)$ of a laminate layer, and the reference system $\left(\begin{array}{lll}x & y & z\end{array}\right)$ of the lamiante. Ply angle $(\theta)$ of a fiber is defined as an angle about $z^{\prime}$ axis.

\section{Fluid-structure interaction interface}

The FSI analysis is based on a time-domain partitioned solution process in which the nonlinear partial differential equation governing the fluid and the structure are solved independently and spatially coupled through the interface between the fluid and the structure. An interface module has been added to the fluid solver to communicate the flow solutions on the three-dimensional wetted surface to and from the structural solver. In current study, an implicit coupling method is used. At each time step the fluid and the structural solvers are called one after the other until sufficient convergence on the displacements on the share boundary are reached in an inner-iteration before advancing to the next time step. Figure 2 illustrates the flow chart of FSI process of current study. . The convergence of a FSI-iteration loop is determined by Eq. (7) where at least two communications between the fluid and the structural solvers are needed,

$$
\Delta s=\frac{1}{N_{\mathrm{CSD}}} \sqrt{\sum_{j=1}^{N_{\mathrm{CSD}}}\left\|\Delta x_{\mathrm{CSD}_{i j}}^{n, \text { fsiloop }_{i j}}-\Delta x_{\mathrm{CSD}_{i j}}^{n, \text { fsilop}_{i j}}\right\|_{i}},
$$

where $N_{\mathrm{CSD}}$ is the number of nodes on the CSD interface mesh, $\Delta x_{\operatorname{CSD}_{i j}}^{n \text {,filop }}$ is the nodal displacement vector on the CSD node $j$ at the time step $n$ and iteration fsiloop. 


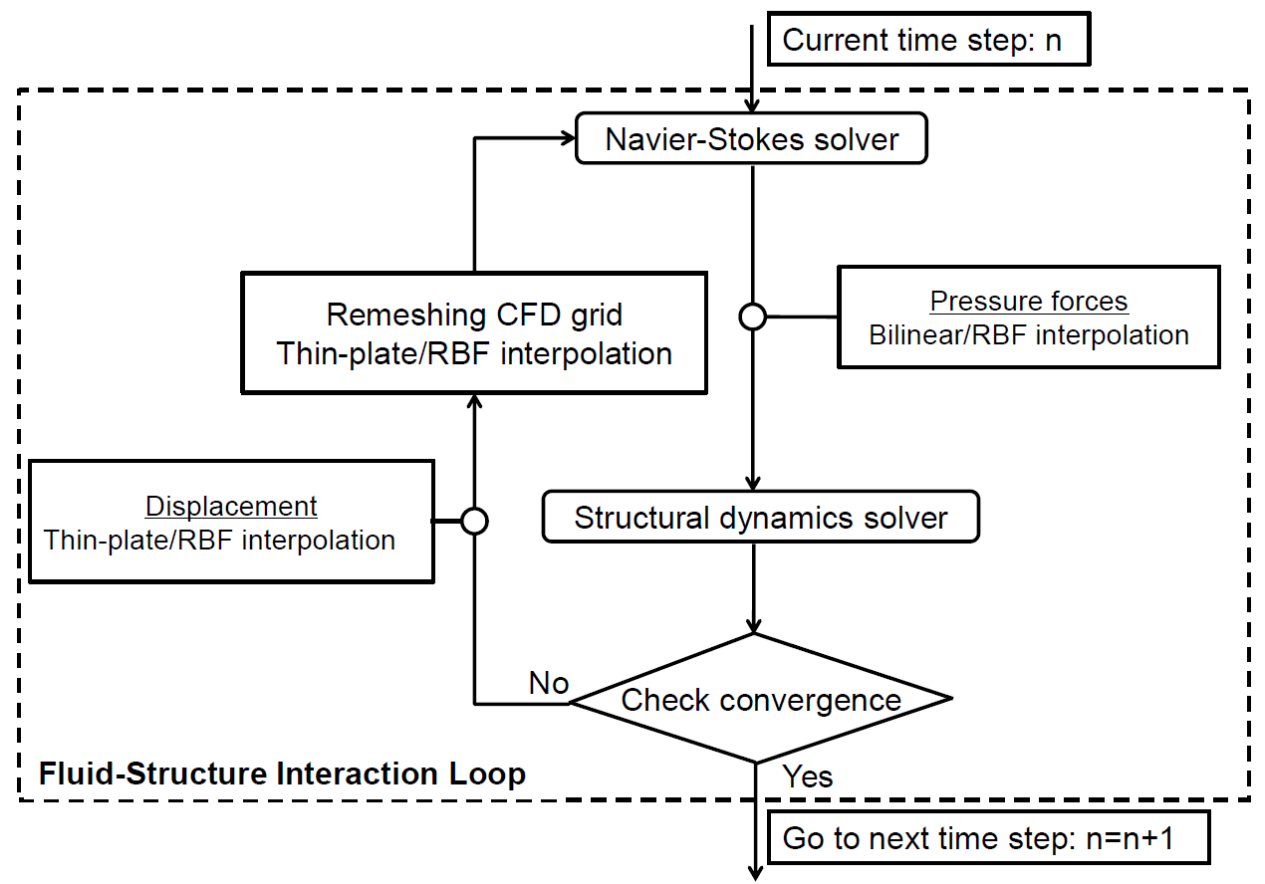

Figure 2 Diagram for time marching loop of fluid-structure interaction.

\section{Dimensional analysis for a Newtonian fluid and isotropic plate}

Dimensional analyses along with flapping wing aerodynamics have been discussed in Refs $[2,3]$. Current study focuses on the interaction between a Newtonian fluid and an isotropic plate. The relevant physical quantities are the density, $\rho_{f}$ and the viscosity, $\mu$, of the fluid; the reference velocity, $U_{\text {ref }}$, of the fluid flow; the half span, $R$, the mean chord, $c_{m}$, and the thickness, $h_{s}$, of the wing geometry; the structural density, $\rho_{s}$, the Young's modulus, $E$, and the Poisson's ratio, $v$, of the wing structure; the flapping amplitude, $\phi_{a}$, the angular flapping frequency, $\omega$, the pitching angle, $\alpha$, of the wing kinematics; and finally the resulting aerodynamic force, $F$. There are 13 variables and 3 dimensions leading to 10 non-dimensional parameters. With $\rho_{f}, U_{\text {ref }}$, and $c_{m}$ as the basis variables the dimensional analysis leads to the following non-dimensional parameters:

\begin{tabular}{|c|c|c|}
\hline Reynolds number & $R e=\frac{\rho_{f} U_{\mathrm{ref}} C_{m}}{\mu}$ & Ratio between the inertial and the viscous forces in the fluids \\
\hline Aspect ratio & $A R=\frac{4 R}{c_{m}}$ & Wing span normalized with the chord \\
\hline Normalized thickness & $h_{s}^{*}=h_{s} / c_{m}$ & Thickness normalized with the chord \\
\hline Density ratio & $\rho^{*}=\rho_{s} / \rho_{f}$ & Ratio between the structural density and the fluid density \\
\hline Poisson's ratio & $v$ & Ratio between the transverse and the axial strain \\
\hline Effective stiffness & $\Pi_{1}=\frac{E h_{S}^{* 3}}{12(1-v) \rho_{f} U_{\mathrm{ref}}^{2}}$ & $\begin{array}{l}\text { Ratio between the elastic bending forces and the } \\
\text { aerodynamic force }\end{array}$ \\
\hline Reduced frequency & $k=\frac{\omega c_{m}}{2 U_{\mathrm{ref}}}$ & $\begin{array}{l}\text { Measure of unsteadiness by comparing the spatial } \\
\text { wavelength of the flow disturbance with the chord }\end{array}$ \\
\hline
\end{tabular}


Strouhal number

(effective) angle of attack

Force coefficient

$$
S t=k A R \phi_{a}
$$

$\alpha$

$$
C_{F}=\frac{F}{1 / 2 \rho_{f} U_{\mathrm{ref}}^{2}\left(2 c_{m}^{2} A R\right)}
$$

Ratio between the flapping speed and the reference velocity

Curvature of the streamlines leading to pressure changes on the wing surface

Aerodynamic force normalized with the dynamic pressure and the wing surface area

If the reference velocity, $U_{\text {ref }}$, is chosen as the velocity scale, inverse of the motion frequency, $2 \pi / \omega$, as the time scale, and the mean chord, $c_{m}$, as the length scale, the governing equations Eqs (2) and (6), and the motion kinematics typically given as

$$
\phi_{s}(t)=\phi_{a} \sin (\omega t)
$$

where $\phi_{s}(t)$ is the instantaneous flapping angle, $\phi_{a}$ the flapping amplitude, and $\omega$ the motion angular frequency, are normalized as, see also Ref 2 ,

$$
\begin{gathered}
\frac{k}{\pi} \frac{\partial u_{i}^{*}}{\partial t^{*}}+\frac{\partial u_{j}^{*} u_{i}^{*}}{\partial x_{j}^{*}}=-\frac{\partial p^{*}}{\partial x_{i}^{*}}+\frac{1}{R e} \frac{\partial}{\partial x_{j}^{*}}\left(\frac{\partial u_{i}^{*}}{\partial x_{j}^{*}}\right) \\
\rho^{*} h_{s}^{*}\left(\frac{k}{\pi}\right)^{2} \frac{\partial^{2} w_{3}^{*}}{\partial t^{* 2}}-\Pi_{1}\left(\frac{\partial^{4} w_{3}^{*}}{\partial x_{1}^{* 4}}+2 \frac{\partial^{4} w_{3}^{*}}{\partial x_{1}^{* 2} \partial x_{2}^{* 2}}+\frac{\partial^{4} w_{3}^{*}}{\partial x_{2}^{* 4}}\right)=-f_{p}^{*} \\
\phi_{s}=\frac{S t}{k A R} \sin \left(2 \pi t^{*}\right) .
\end{gathered}
$$

The non-dimensional variables that appear in the governing equation for the wing structures apart from the reduced frequency are $\Pi_{1}, \rho^{*}$, and $h^{*}$. The instantaneous lift and thrust coefficients are defined as

$$
\begin{gathered}
C_{L}=\frac{L}{\frac{1}{2} \rho_{f} U_{\mathrm{ref}}^{2}\left(2 c_{m}^{2} A R\right)}, \\
C_{T}=\frac{T}{\frac{1}{2} \rho_{f} U_{\mathrm{ref}}^{2}\left(2 c_{m}^{2} A R\right)},
\end{gathered}
$$

where $S$ is the planform area of the wing, $L$ is the lift, and $T$ the thrust, such that for the resulting force, $F_{i}$, we have $L=F_{1}$ and $T=-F_{2}$.

\section{Results and Discussions}

\section{A. Remeshing: rigidly moving flat plate in a square box}

To assess the performance in terms of the mesh qualities, and domain scalability of the RBF interpolation remeshing method, two test cases previously reported by Stein et al. ${ }^{22}$ and Smith and Wright ${ }^{23}$ are computed. The initial mesh consists of a computational domain in a square shape with length of two and a two percent thick flat plate of chord length one in the center of the domain, see Figure 3. In all solutions shown in the current study the new mesh is obtained after one time step. For the RBF the Wendland's C2 polynomial is used with a support radius of 2.0. The linear solver converged to the imposed absolute tolerance level of $1.0 \times 10^{-30}$. In the first case the flat plate is rotated $60 \mathrm{deg}$ around its mid-chord point of the plate. The order of this angle corresponds to typical flapping angle amplitudes ${ }^{1}$. In the second case the flat plate translates half chord length upwards. Note that the mesh is threedimensional with unit span length with one cell in spanwise direction to simulate two-dimensional fluid flow in a three-dimensional fluid solver. This doubles the number of grid nodes from the two-dimensional situation. 


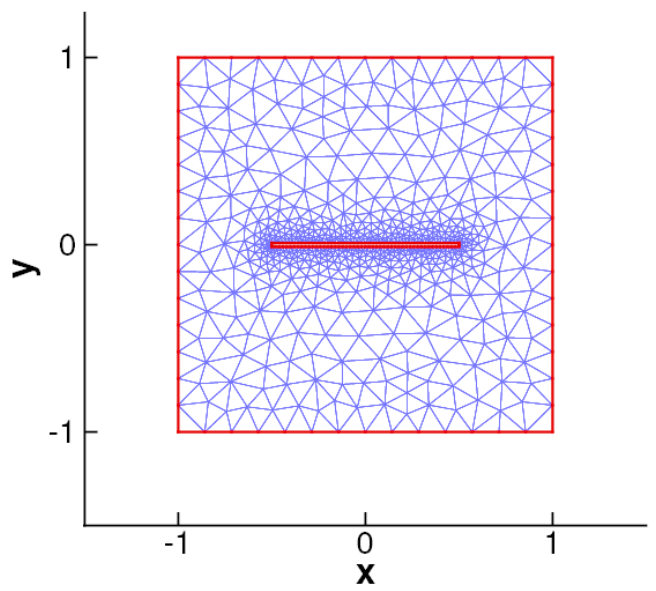

Figure 3 Initial mesh of a two percent thick flat plate in a square domain. Total number of cells, nodes, and boundary nodes are 1224, 1384, and 520, respectively.

Two grid measures are computed to assess the mesh qualities: non-smoothness, which measure the cell volume change in space, defined as

$$
\text { non-smoothness }=\sqrt{\frac{1}{A}\left(\frac{\partial V}{\partial x_{1}}\right)^{2}+\left(\frac{\partial V}{\partial x_{2}}\right)^{2}},
$$

where $V$ is the cell volume, $A$ is the cell area. To measure the skewness of a cell the following quantities are determined

$$
\text { skewness }=1-\frac{\text { length of shorter face diagonal }}{\text { length of longer face diagonal }}
$$

For both mesh quality metrics high values would result in worse accuracy of the fluid flow solutions and small values are desired. The domain scalability of the current remeshing method with respect to the mesh size is measured by refining the initial mesh by multiplying the number of nodes on the boundary faces by $N=2,4,8$, and 16, see Table 1.

Table 1 Number of cells, number of nodes, and number of boundary nodes depending on the multiplication factor $N$.

\begin{tabular}{cccc}
\hline \hline$N$ & Number of cells & Number of nodes $\left(N_{v}\right)$ & Number of boundary nodes $\left(N_{b}\right)$ \\
\hline 1 & 1224 & 1384 & 520 \\
2 & 3272 & 3592 & 1040 \\
4 & 10142 & 10782 & 2080 \\
8 & 35896 & 34616 & 4160 \\
16 & 127496 & 130056 & 8320 \\
\hline \hline
\end{tabular}

As the RBF interpolation remeshing method acts on the distances, the cells near the flat plate being more influenced by the motion of the flat plate than the cells away, the cells around the flat plate move almost rigidly, see Figure 4. Figure 5 shows the non-smoothness and the skewness of the cells on the initial mesh and the resulting mesh under the rotation and translation for the baseline case $(N=1)$ and the maximally refined case $(N=16)$. The focus is given to how well the initial mesh qualities are preserved under remeshing process. In small region between the edge of the flat plate and the outer boundary of the computational domain both non-smoothness and skewness suffer due to the shearing effect between the moving flat plate and the non-moving outer boundary. However, overall, both the nonsmoothness and the skewness near the flat plate are well preserved. On the refined mesh the cells are so small that the smoothness remains intact, which was also observed for $N=2$, 4, and 8. Regarding the skewness, again due to the shearing behavior, the skewness increases between the flat plate and the boundaries of the computational domain, still the mesh qualities remain well near the flat plate which is important to capture smaller near-wall flow phenomena accurately. 


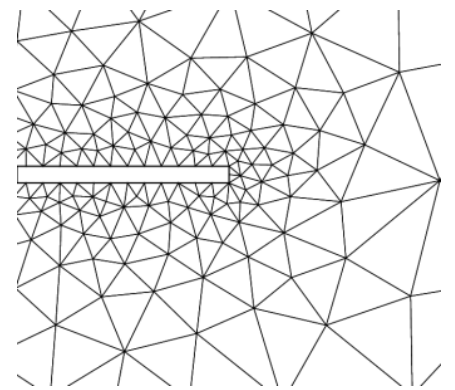

(a) initial distribution

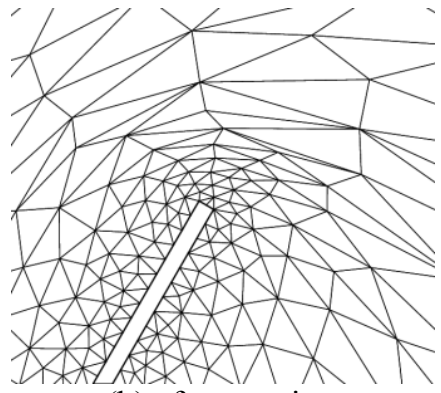

(b) after rotation

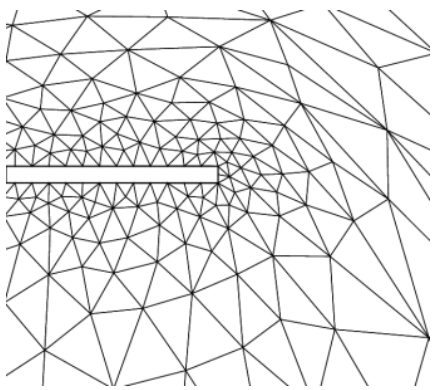

(c) after translation

Figure 4 Mesh distribution near the trailing-edge of the flat plate on (a) the initial grid, (b) after rotation, and (c) after translation for the baseline grid $(N=1)$.

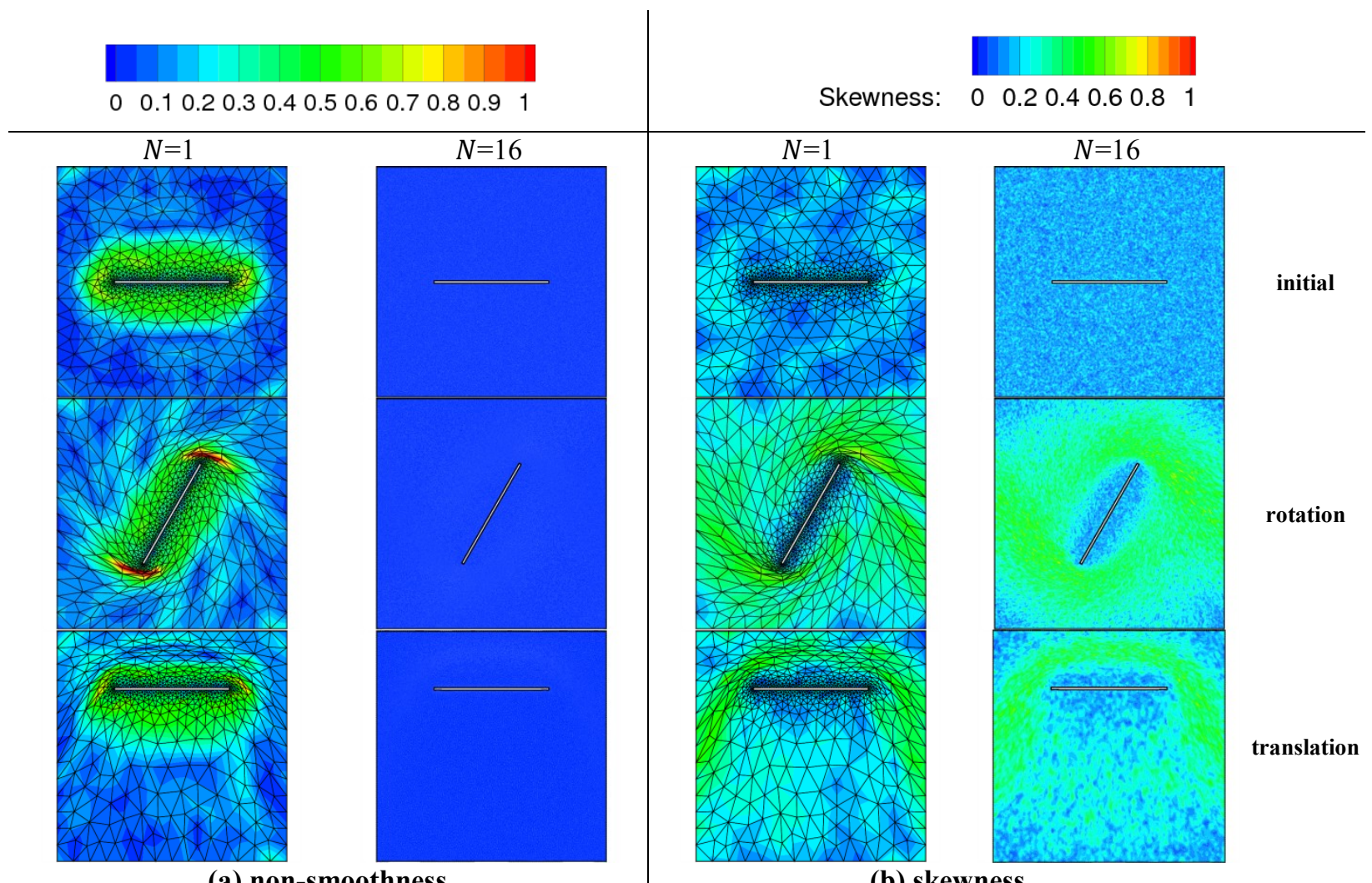

(a) non-smoothness

(b) skewness

Figure 5 Non-smoothness and skewness of the cells on the initial mesh and the rotated mesh for the mesh scalability factors $N=1$ and 16 of a flat plate in a non-moving square shaped outer boundary under the rotational and translational motions. Note that on the $N=16$ grid the mesh distribution is not shown for visibility purposes.

The domain scalabilities are shown in Figure 6, which is defined as

$$
\text { Domain scalability }=\frac{\text { Time }_{N}}{\text { Time }_{N=1}} .
$$

On a single processor the domain scalability is assessed for the rotation and translation cases. The complexity of the linear solver scales with $N_{b}^{2}$ and the RBF evaluation process of Eq. (3) with $N_{v} \cdot N_{b}$ where $N_{v} \sim N_{b}^{2}$. If the number of boundary nodes is doubled, i.e. $N=2$, then the domain scalability would be $O\left(N^{2}\right)$, which is confirmed in Figure 6 . 


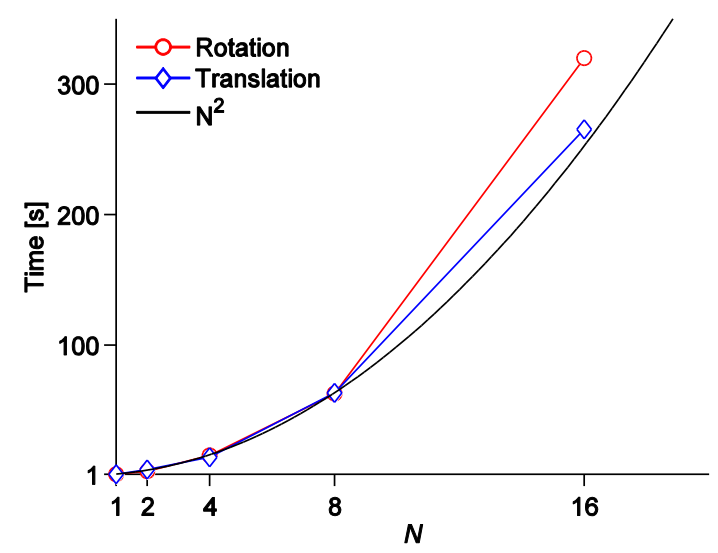

Figure 6 Domain scalabilities of a flat plate in a non-moving square domain under rotation and translation.

Finally, the influence of the support radius, $r$, on the resulting computational time and mesh qualities are assessed by varying the radius from 2 to 1 and 3 on the $N=8$ grid using single processor for the translational motion. For the compact radial basis functions, such as Wendland's $C^{2}$ function, Eq. (4), used here, the support radius sets the radius influence by setting the radial basis function value to zero if the distance between two points becomes larger than the radius. Table 2 shows the computational time taken for different support radius. By reducing the support radius from $r=2$ to $r=1$ the computational cost reduces because the coupling matrix $\mathbf{M}_{\mathrm{b}, \mathrm{b}}$ in Eq. (5) is sparser. On the other hand, increasing $r$ to 3 introduces more coupling between the boundary nodes and results in $23 \%$ increase in computational time. However, for smaller $r$, the influence of the moving nodes is propagated only for smaller distances and the resulting mesh distortions remain close to the flat plate. This behavior is shown in Figure 7 where the skewness is shown for $r=1,2$, and 3. For $r=1$ the deformation occurs close to the flat plate and the cells are more clustered, while for $r=3$ the skewness is smoothened out over larger area.

Table 2 The computational time in components for the translating flat plate in a square domain on the $N=8$ mesh using single processor.

\begin{tabular}{cccc}
\hline \hline$r$ & Linear solve (s) & Applying Eq. (3) (s) & Total time (s) \\
\hline 1 & 0.30 & 6.1 & 6.4 \\
2 & 0.76 & 12 & 13 \\
3 & 1.8 & 14 & 16 \\
\hline \hline
\end{tabular}

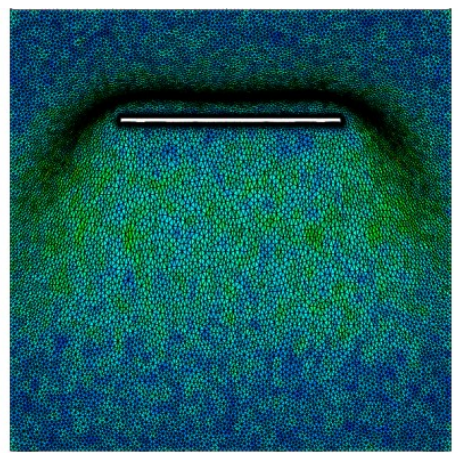

(a) $r=1$

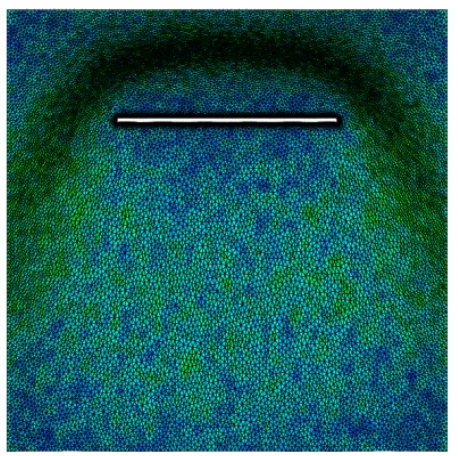

(b) $r=2$

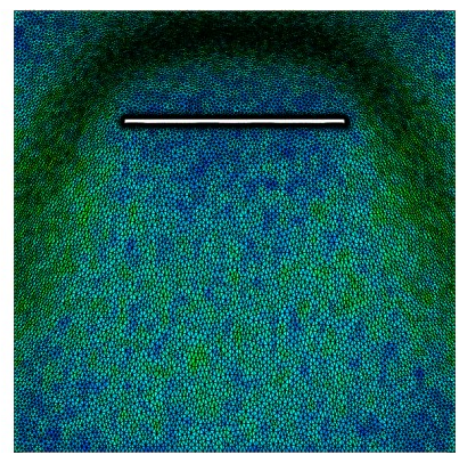

(c) $r=3$

Figure 7 Skewness of the cells on the $N=8$ grid of a flat plate in a non-moving square shaped outer boundary under the translational motion. Black lines represent the mesh distribution. 


\section{B. A rigid rectangular wing prescribed with plunging excitation in free-stream at $R e=3.0 \times 10^{4}$ and $S t=0.20$}

A rectangular wing prescribed with prescribed pure plunging excitation is simulated and compared with previous experimental study ${ }^{9}$. The computational result is obtained using the parallel version NS solver with radio basis function interpolation remeshing method. A schematic of the computational setup is the same as Refs. 4 and 8 and is shown in Figure 8. The computational wing model has semi-span length of $0.3 \mathrm{~m}$ and a chord of $c_{m}=0.1 \mathrm{~m}$. A C-O type grid around a NACA0012 wing of aspect ratio 6 is used (Figure 8 (b)). The number of grid points is $120 \times 56 \times 60$ in the tangential, radial, and spanwise directions, respectively. The grid refinement and time step sensitivity analyses have been performed to identify a suitable grid configuration in terms of the number of points, and the time step increment in Ref. 4.

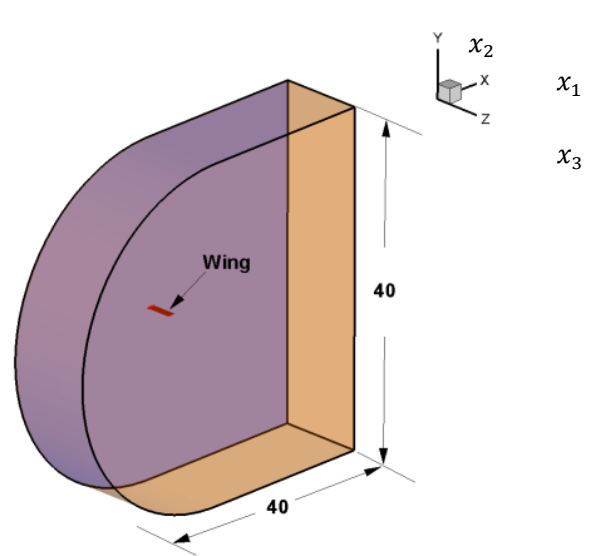

(a) Overview of grid topology and domain size

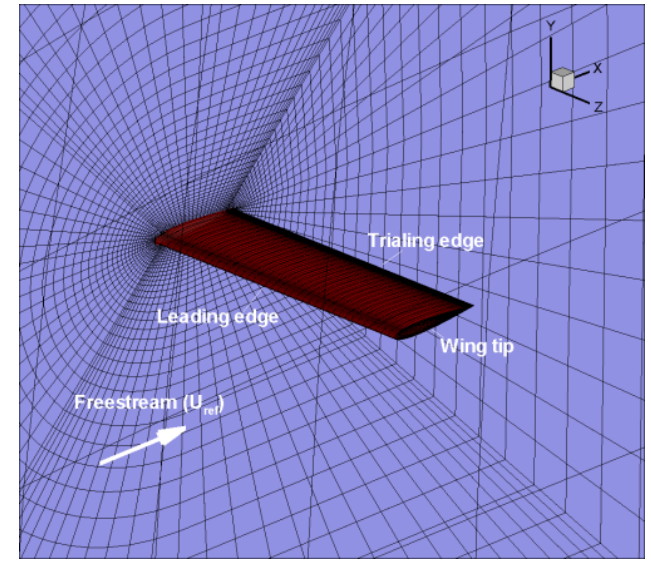

(b) wing grid

Figure 8 Computational setup for a rectangular wing prescribed with pure plunging excitation. Purple plane indicates symmetrical plane. (a) Overall grid topology, (b) near wing. The direction of free-stream is $x_{1}$-axis. The wing plunges in $x_{2}$ direction.

As to the prescribed wing motion, the leading edge of the wing root is actuated by a sinusoidal plunge displacement profile. The amplitude and frequency of plunging motion is $0.175 c_{m}$ and $1.74 \mathrm{~Hz}$. For the boundary conditions at the inlet, the free-stream velocity of $0.3 \mathrm{~ms}^{-1}$ is prescribed, and for the outlet, the pressure is set to zero. A no-slip condition on the flow velocity is assigned to the wing surface. The nodes on the outer boundary surfaces are fixed in space and time. For the support radius of the radio basis function 0.5 is chosen which is of the same order of magnitude but larger than the half span length of 0.3 . Because both the boundary nodes on the outer boundary surfaces with zero displacements and the prescribed wing displacements construct the coupling matrix $\mathbf{M}_{\mathrm{b}, \mathrm{b}}$ in Eq. (5) the computational cost to remesh consist about $55 \%$ of the total computational time. A simple method to increase the computational efficiency is presented in the section III A. The computation is run for approximately 10 cycles (i.e. 5100 time steps) using 16 Intel ${ }^{\circledR}$ Xeon ${ }^{\circledR}$ E5540 (8M Cache, $2.53 \mathrm{GHz}, 5.86 \mathrm{GT} / \mathrm{s}$ Intel ${ }^{\circledR}$ QPI) processors. Per time step, the linear solver took 20 seconds to solve the linear system for the remeshing, and 17 seconds on the slowest processor to apply Eq. (3). 


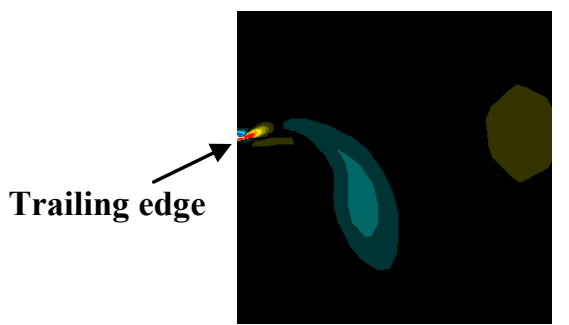

(a-1) at mid-span: current study
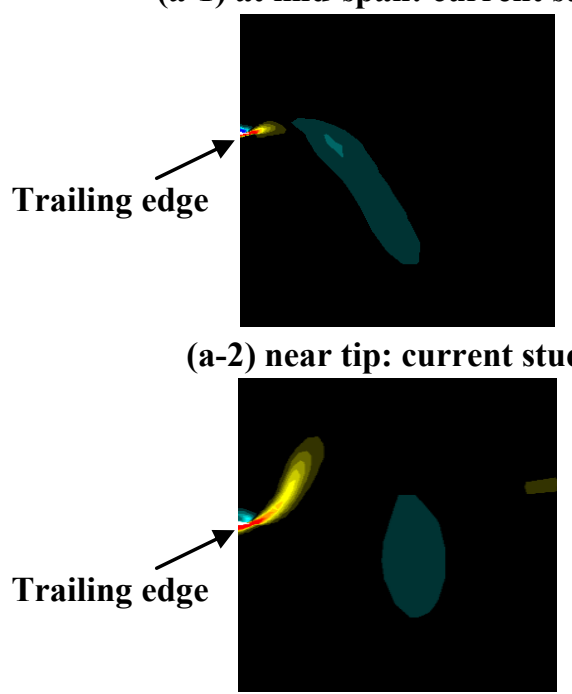

(a-3) at mid-span: current study

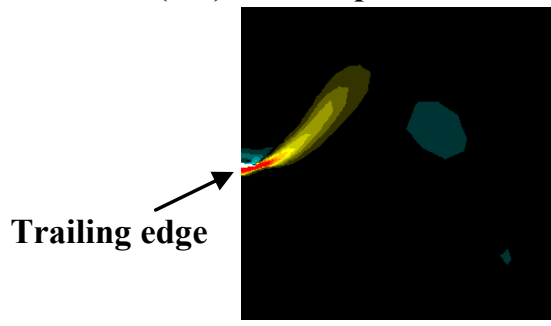

(a-4) near tip: current study

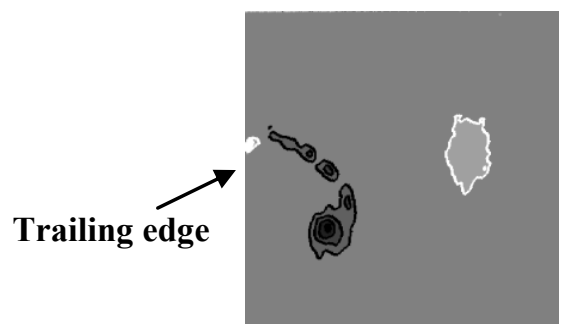

(b-1) at mid-span: experiment

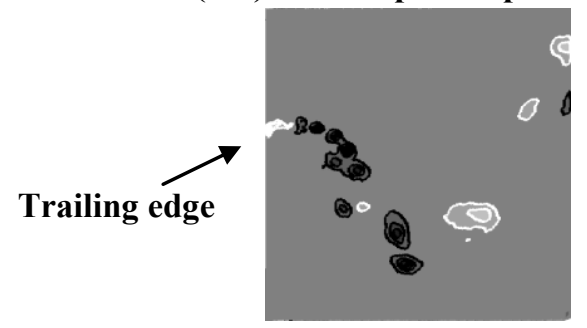

(b-2) near tip: experiment

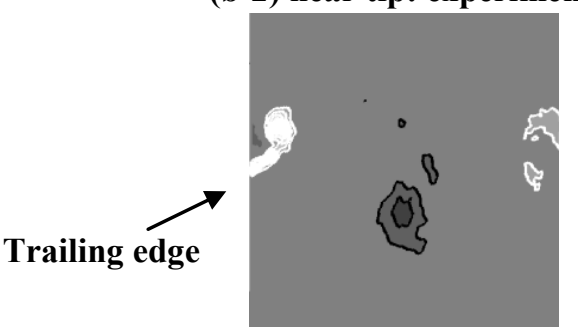

(b-3) at mid-span: experiment ${ }^{9}$

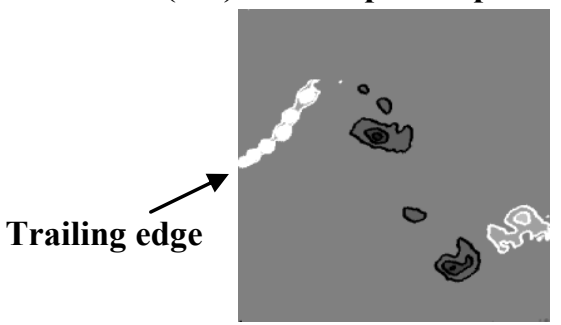

(b-4) at near tip: experiment ${ }^{9}$

Figure 9 Comparison of spanwise vorticity contours in two selected wing sections (i.e. mid-span and $93 \%$ span) between current study and experiment ${ }^{9}$ at the beginning (a-1,2 and b-1,2) and middle (a-3,4 and b-3,4) of the downstroke (i.e. $t / T=$ 0.0 and 0.25$)$. The wing is moving downward. $\left(R e=3.0 \times 10^{4}\right.$ and $\left.S t=0.20\right)$. Vorticity is normalized by $\frac{c_{m}}{U_{\text {ref }}}$.

Figure 9 illustrates a comparison of spanwise vorticity contours at two selected wing sections (mid-span, near tip) between the current study and experimental result ${ }^{9}$ at the beginning and middle of downstroke. Overall, the large scale flow structure computed by the current numerical framework is comparable, although, experimental result shows a cluster of smaller vortices near the wing. Figure 10 shows a comparison of aerodynamic forces between the current study and experiment ${ }^{9}$. It is found that the time histories of thrust and lift coefficient of the current study show good agreement with the previous numerical and experimental studies. 


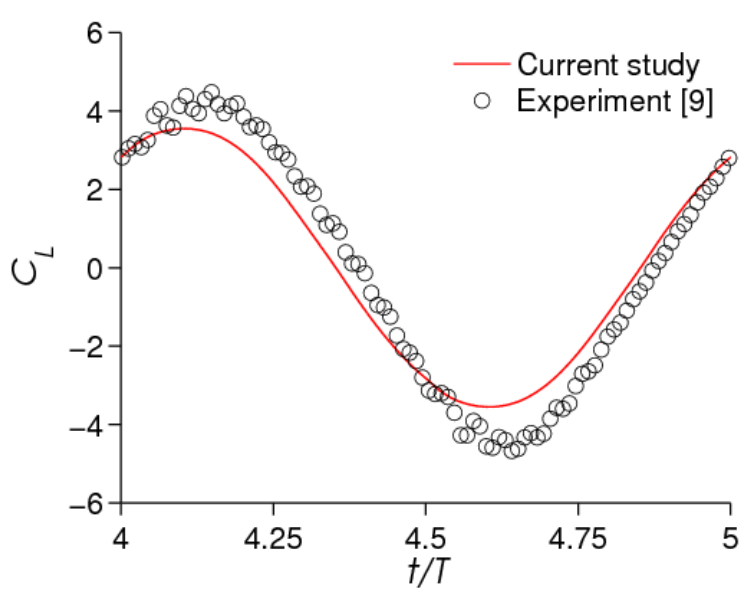

(a) Lift coefficient

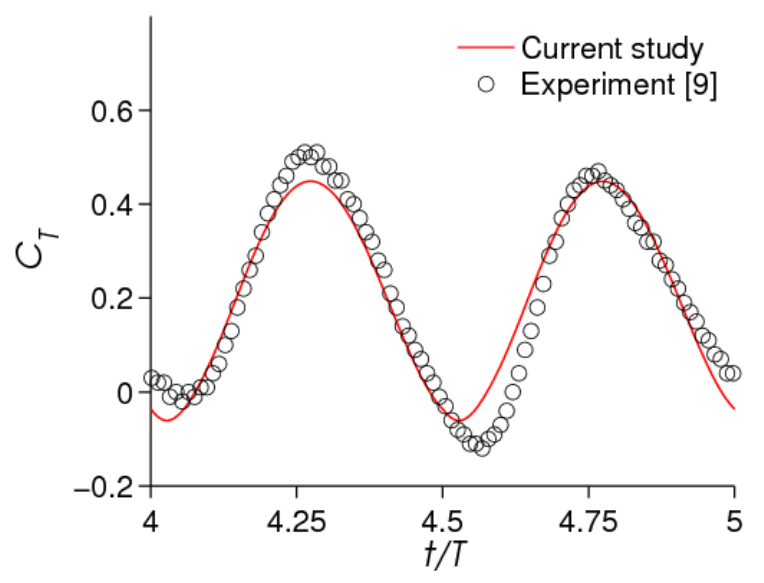

(b) Thrust coefficient

Figure 10 Time histories of lift and thrust coefficient for a rectangular wing prescribed with pure plunging excitation at $R e=3.0 \times 10^{4}$ and $S t=0.20$. Solid red line indicates the result of current study. Open circles are from the result of experimental study9.

\section{An anisotropic elliptic wing prescribed with single DOF flap rotation in vacuum}

In order to be able to investigate effects of anisotropic wing on aerodynamic force generation, it is necessary to validate a composite wing structure model first. In the current study, a composite wing structure model is implemented based on the classical laminated theories ${ }^{39}$. In this section, structural dynamic response of composite flapping wing in vacuum will be compared with the experimental data ${ }^{12}$ for validation purpose.

Figure 11 shows design of anisotropic wing and setup of computational composite wing models. The wing planform is Zimmerman elliptic, the aspect ratio is 7.65 , and the mean chord length of the wing is $19.6 \times 10^{-3} \mathrm{~m}$. The wing are built with unidirectional carbon fiber to form the skeleton and Capran ${ }^{\circledR}$ as the membrane. As shown in Figure 11, two simulated wings are made of the same structural layout: reinforced leading edge, root triangle, and reinforced chordwise battens. There is no reinforcement of the trailing edge. Figure 11 (a) presents layup schemes: the lines represent the number of layers for layup - leading edge varies from 3 to 1 layers of unidirectional carbon fiber and chordwise batten varies from 2 to 1 layers. Therefore, the wings are named $\mathrm{L}(\mathrm{m}) \mathrm{B}(\mathrm{n})$ with $\mathrm{L}$ for leading edge, $\mathrm{m}$ for number of layers in leading edge, $\mathrm{B}$ for chordwise batten and $\mathrm{n}$ for chordwise layer numbers More detail of the wing structures and the experimental setup can be found in Ref. [11]. In the current study, L2B1 and L3B1 are considered and the total number of structural mesh is 767 elements. The prescribed flapping excitation is given at the root triangle and its profile is sinusoidal. The structural and geometrical information of carbon fiber and Capran ${ }^{\circledR}$ membrane are given in Table 3. For simple purpose, membrane-bending coupling effect of stiffness matrix is ignored. Generalized- $\alpha$ method is utilized to integrate in time. Flapping amplitude is \pm 35 deg and flapping frequency is $25 \mathrm{~Hz}^{12}$.

Figure 12 shows a comparison between the computed results and experimental data in terms of vertical tip deflection and vertical tip location. The vertical tip deflection is measured with respect to the undeformed wing configuration at corresponding flapping angle, and the vertical tip location is determined with respect to inertial frame as illustrated in Figure 12 (a). Both quantities are normalized by the mean chord length. The experimental data are acquired during one flapping cycle. The instantaneous computed wing tip deflections of L2B1 and L3B1 show overall agreement with the experimental data ${ }^{12}$. It is found that the maximal tip deflection of L2B1 doubles compared to that of L3B1. Also trajectory of tip deflection of L2B1 generates considerably larger enclosed area than that of L3B1. These observations indicate that adding one more layer of reinforced leading edge batten has significantly impacts on the structural dynamic response. Regarding the vertical wing tip locations, the computed results of L2B1 and L3B1 show good agreement with the experimental data ${ }^{12}$. Compared to vertical tip deflection, the vertical wing tip location is not as much affected by the change of number of reinforced leading edge batten. Still, the influence of wing deformation is observed when the wing accelerates or decelerates. In addition to these observations the computed results show cycle to cycle variations. Furthermore, due to mechanical constraints, the flapping motion in the experiment doesn't exactly follow a sinusoidal pattern. These issues are being investigated and will be further clarified in the future. 

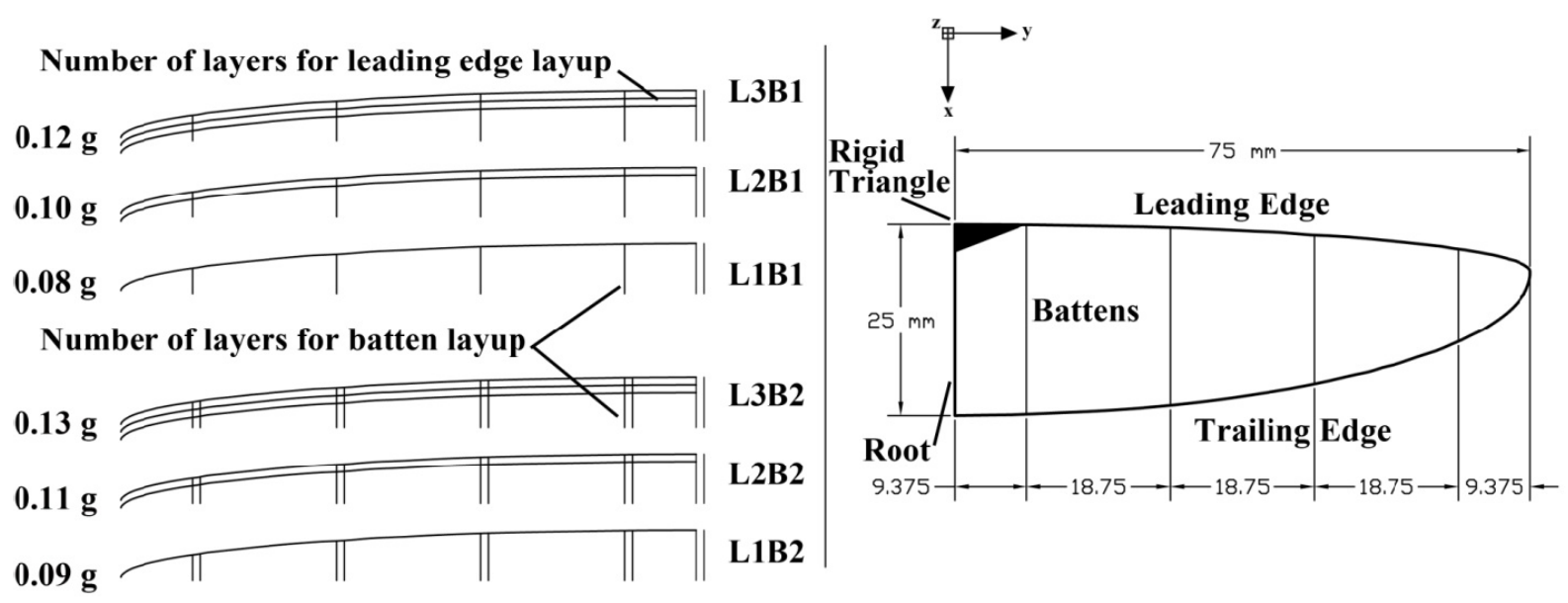

(1) Schematic of reinforcement layout of the anisotropic wing [12]

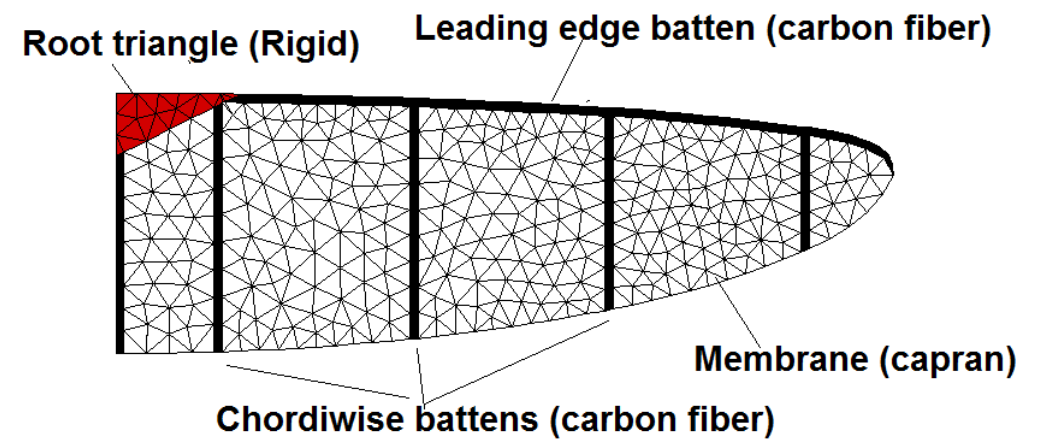

(2) Computational model (767 elements)

Figure 11 Design of anisotropic wings and setup of computational composite wing models.

Table 3 Material properties and geometrical information of structure ${ }^{40}$

\begin{tabular}{ll}
\hline Carbon fiber & $E_{11}=233 \mathrm{GPa}, E_{22}=23.1 \mathrm{GPa}, G_{12}=15.5 \mathrm{GPa}, v_{12}=0.05$, \\
& $\rho_{s}=1740 \mathrm{~kg} / \mathrm{m}^{3}, h_{s}=1.0 \times 10^{-4} \mathrm{~m}$, \\
& $\theta=0$ deg. for the leading edge batten; $\theta=90$ deg for the chordwise batten \\
\hline \hline Capran® Membrane & $E=2.76 \mathrm{GPa}, v=0.489, \rho_{s}=1384 \mathrm{~kg} / \mathrm{m}^{3}, h_{s}=1.5 \times 10^{-5} \mathrm{~m}$ \\
\hline
\end{tabular}

Figure 13 shows instantaneous wing configuration of computed results at the ends of strokes. It can be seen the local deformation is mostly due to the anisotropic wing structure constructed with a membrane and carbon fibers. Since approximately $80 \%$ of the wing area consists of a membrane, resulting bending motion of the leading edge batten affects the deformation of the membrane significantly. At the end of the downstroke, larger vertical wing tip deflection of L2B1 induces larger twisting motion of the wing (see Figure 13 (a-1) and (b-1)). At the beginning of the downstroke, the L3B1 shows more twist than L3B1 (Figure 13 (a-2) and (b-2)) because of weaker influence of the leading edge batten to the membrane. These observations suggest that the deflection and movement of leading edge batten are the main driver of the wing motion, whereas the influence of deformations in other parts is observed at the end and start of the strokes. Therefore three-dimensional wing deformation becomes very complex when the wing accelerates or decelerates. 


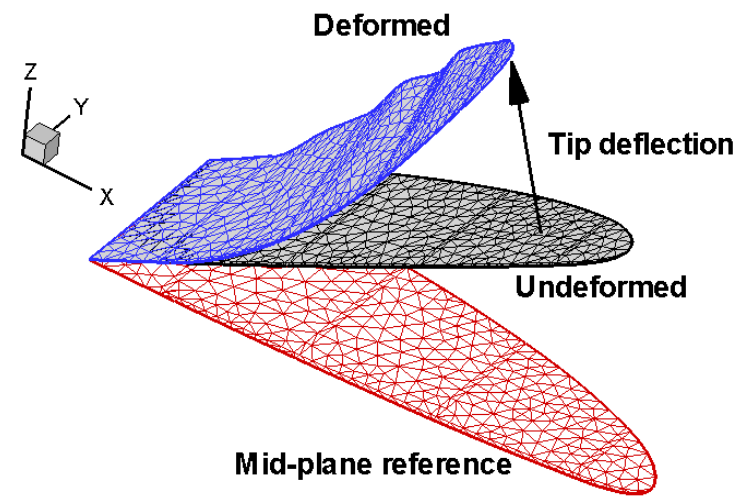

(a) Schematic of wing tip deflection and coordinate system
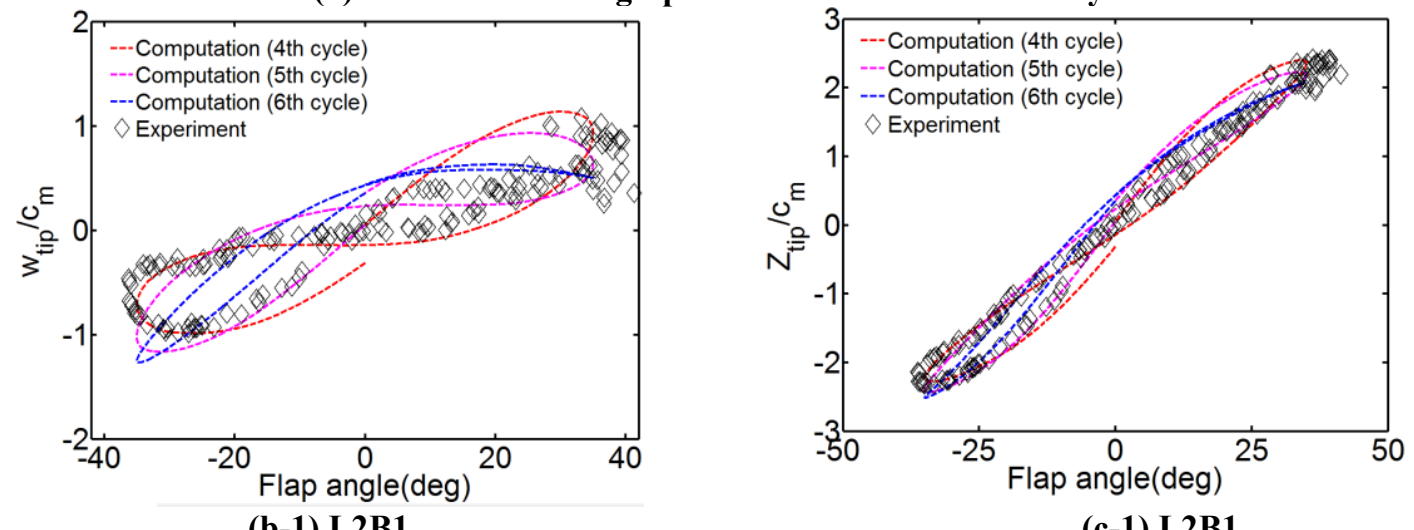

(b-1) L2B1
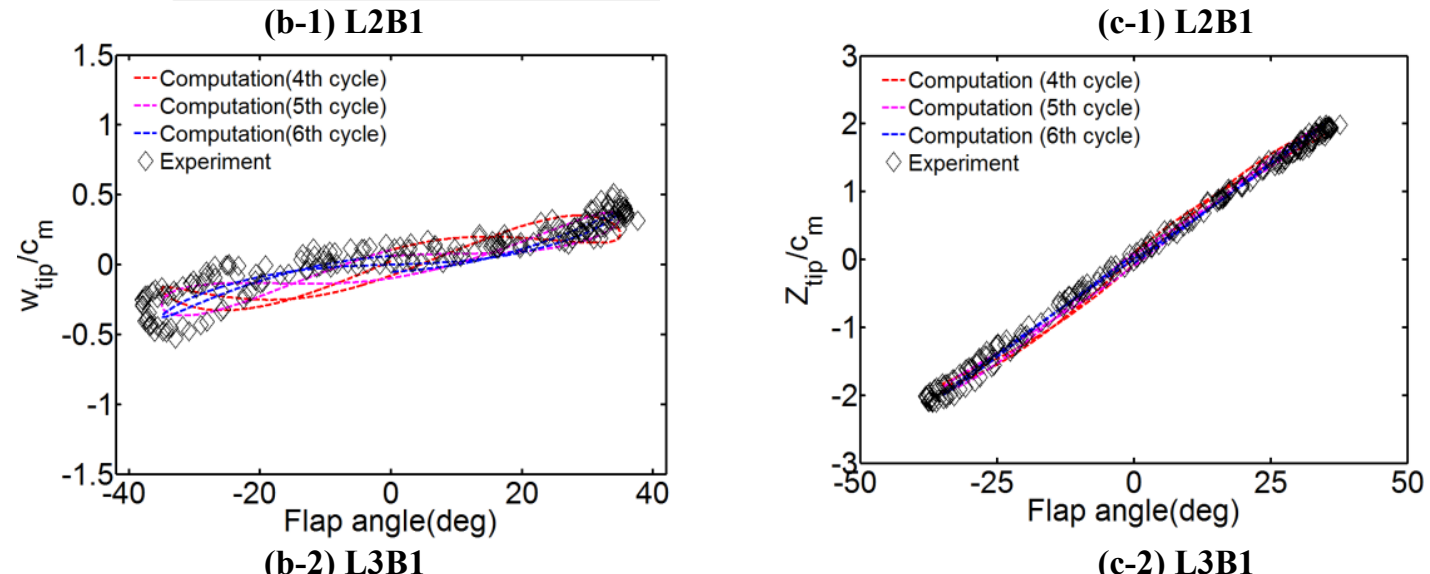

Figure 12 Wing tip deflection and location of L2B1 and L3B1 over flapping cycle. Fourth, fifth, and sixth cycle of prescribed flapping excitation are shown. 

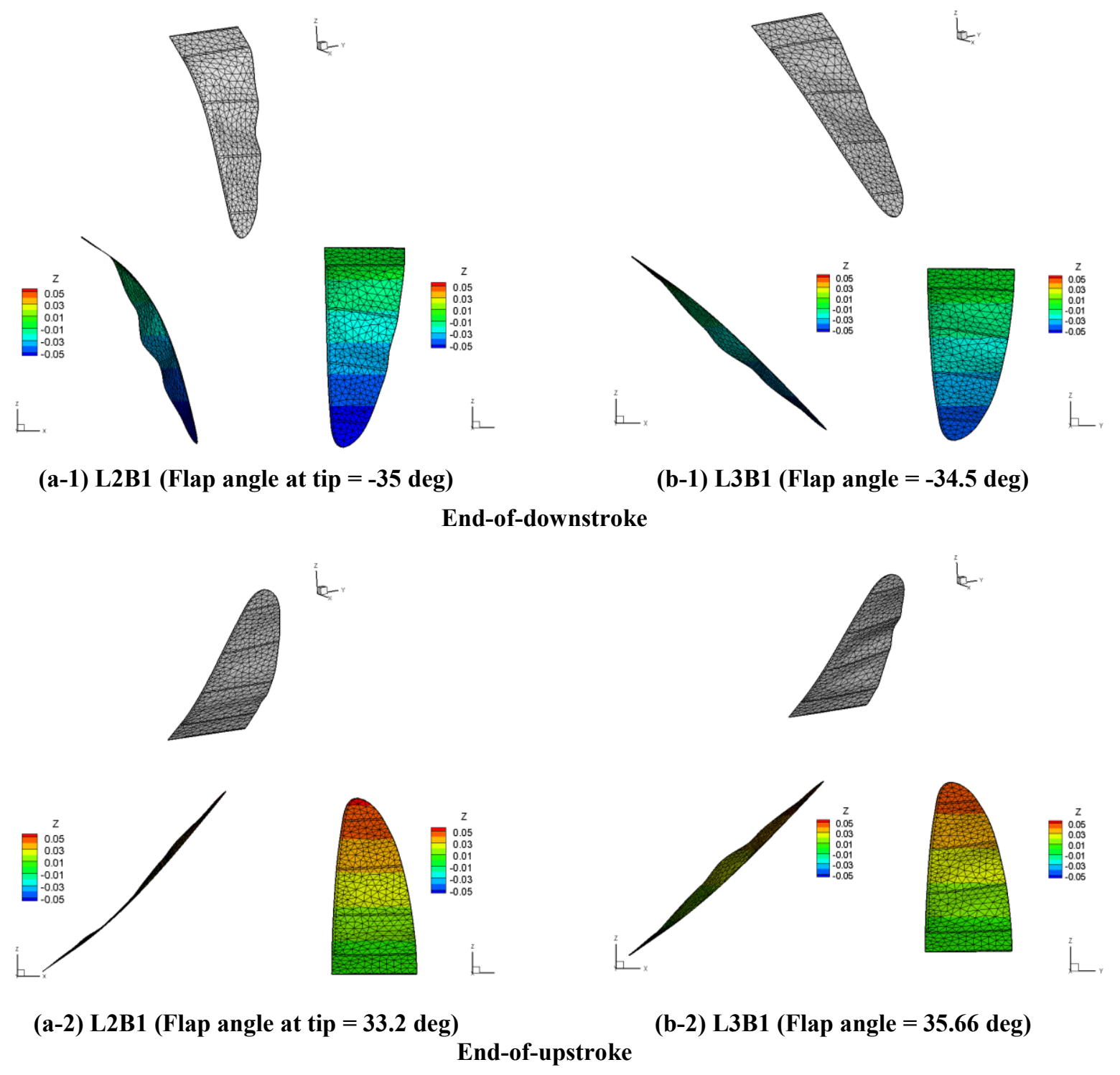

Figure 13 Wing deformation and vertical displacement of L2B1 and L3B1 in vacuum at end of strokes. Contour indicates vertical displacement with respect to inertial frame.

\section{A Zimmerman elliptic wing prescribed with pure flap rotation in hovering at $R e=1.5 \times 10^{3}$ and $k=0.56$ 1. Rigid wing}

A hovering rigid Zimmerman elliptic wing prescribed with pure flapping excitation $R e=1489$ and $k=0.56$ is simulated. The computational result is obtained using the parallel version NS solver with radial basis function interpolation remeshing method. The Zimmerman planform, shown in Figure 14, flaps in a single degree of freedom motion in still air.

A mixed structured and unstructured grid was used, which is the same as used in Ref. 18: the number of points in the converged grid configuration is 0.5 million approximately. The computational setup is presented in Figure 15 and a summary of key parameters of this case are furnished in Table 4. The motion is excited at the rigid square at the leading edge of the wing with a sinusoidal motion; see Eq. (8). For the boundary conditions for the outlet, the pressure is set to zero. A no-slip condition on the flow velocity is assigned to the wing surface. The nodes on the symmetry plane, the purple colored plane in Figure 15, are set to zero displacements. To assess the effect of including the boundary nodes on the outer boundary surface in the remeshing system setup, in contrast to the results presented in Section A, these boundary nodes are set to move freely in space and time. The radial basis function 
support radius is 0.075 , the half span length. By letting the outer boundary surfaces freely to move and taking smaller support radius the computational efficiency is seen to be $20 \%$.

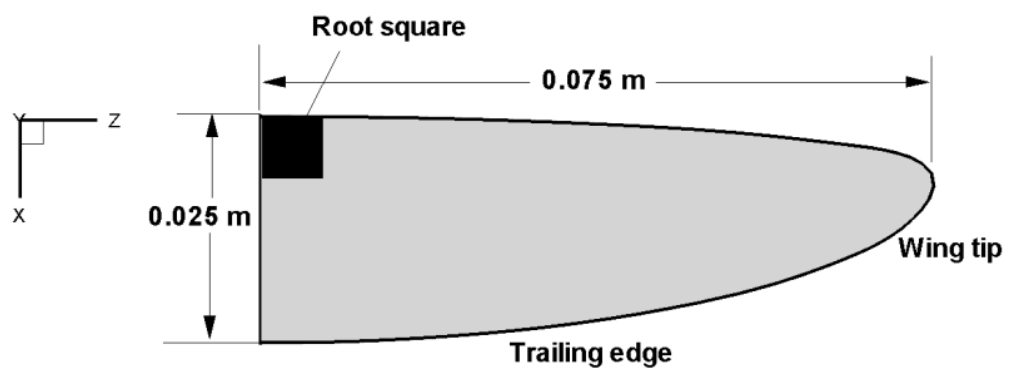

Figure 14 Geometry of a Zimmerman planform.

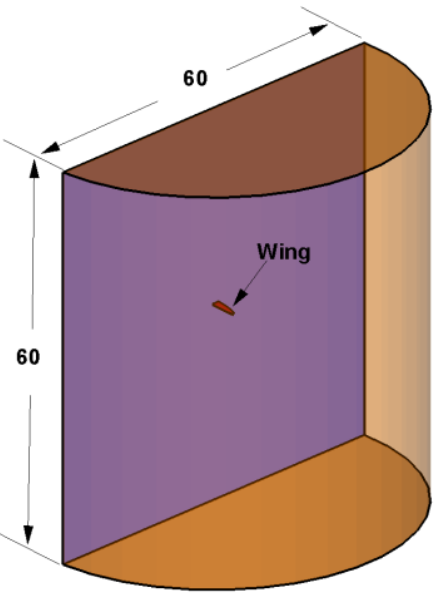

(a) Overview of grid topology and size
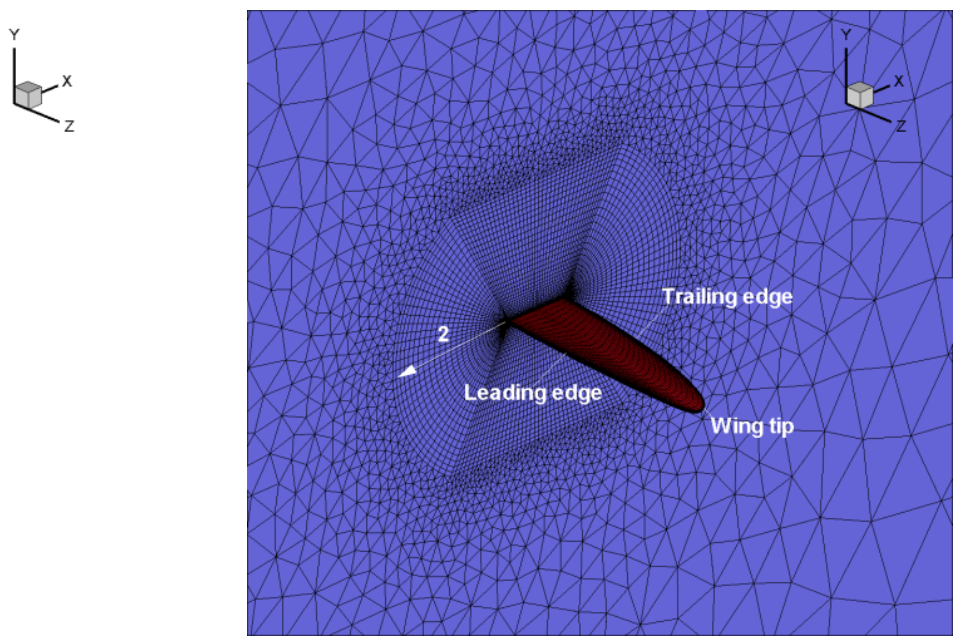

(b) wing grid

Figure 15 Computational setup for a flapping Zimmerman elliptic wing. (a) Overview of grid topology and size; (b) Near the wing.

Table 4 Dimensional and dimensionless parameters for a Zimmerman elliptic wing prescribed with single DOF flap rotation.

\begin{tabular}{lclc}
\hline \hline \multicolumn{1}{c}{ Parameter } & Value & \multicolumn{1}{c}{ Dimensionless Parameter } & Value \\
\hline Semi-span at quarter chord $[\mathrm{m}]$ & 0.075 & Chord-based Reynolds number & 1489 \\
Mean chord length $[\mathrm{m}]$ & 0.0196 & Reduced frequency & 0.56 \\
Flow velocity $[\mathrm{m} / \mathrm{s}]$ & 1.1 (mean tip speed) & Aspect ratio & 7.65 \\
Air density $\left[\mathrm{kg} / \mathrm{m}^{3}\right]$ & 1.23 & & \\
Flapping amplitude $[\mathrm{deg}]$ & 21 & & \\
Flapping frequency $[\mathrm{Hz}]$ & 10 & & \\
\hline \hline
\end{tabular}

Figure 16 shows time history of lift and drag coefficients for first three flapping cycle. It can be seen that the drag coefficient is almost zero because of the wing kinematics considered in this study (i.e. symmetrical flapping motion and no variation of angle of attack in time). Instantaneous lift peak is observed at early downstroke. Both curve profiles of instantaneous lift and drag coefficient are comparable to the results of the stiffest wing case presented in Ref. [18]. Figure 17 presents flow fields around the elliptic Zimmerman wing undergoing pure flapping motion at the beginning and middle of downstroke. Three-dimensional vortical structures are visualized by isovorticity magnitude and two-dimensional vortical structure is plotted by spanwise vorticity contours. Clearly, there is three-dimensional vortical structure around the wing and an interaction between the flapping wing and vortex 
wake. Interestingly, it can be seen that ring-shaped like wake structure beneath the wing (see Figure 17 (a)) that generated by previous stroke. Similar three-dimensional vortical structures were observed in flow structures generated by biological flapping flyers ${ }^{1,2,41}$. Moreover, it is found that leading and trailing edge vortices interact with shed vortices generated in previous stroke (see Figure 17 (b)).

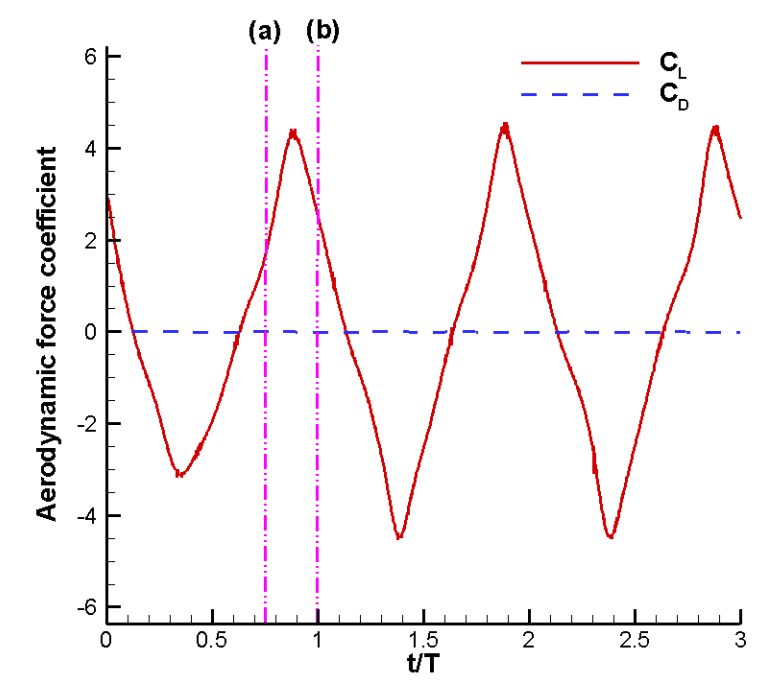

Figure 16 Time history of lift and drag coefficient for first three flapping cycle. Solid red and dashed dotted lines are lift and drag coefficient. Flow fields around the wing at time instants (a) and (b) will be presented in next figure.

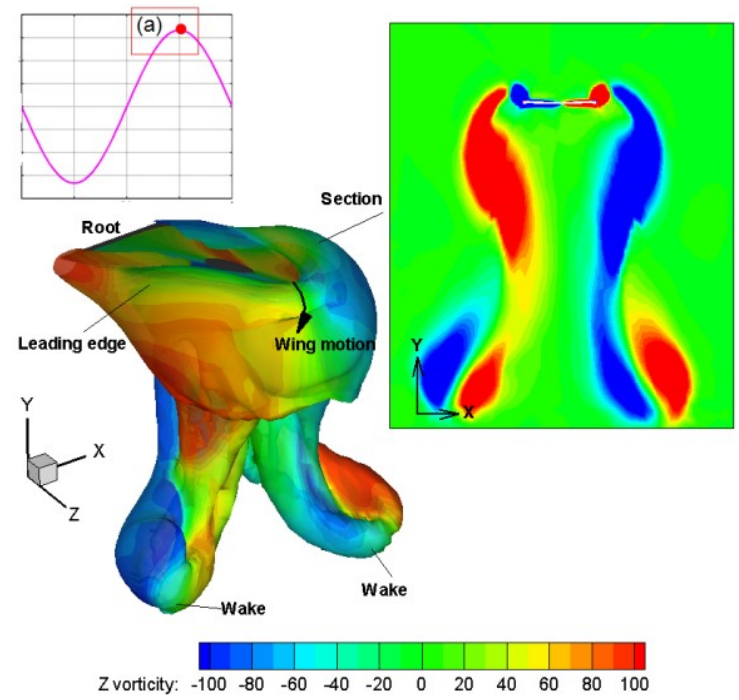

(a) begging of downstroke

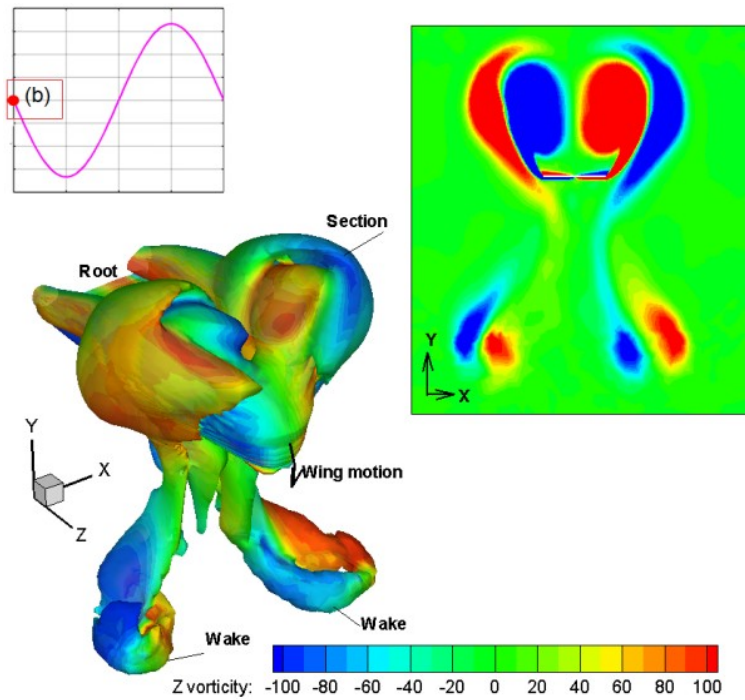

(b) middle of downstroke

Figure 17 Flow visualization around the Zimmerman elliptic wing undergoing pure flapping motion. Line plots presented in left top indicate the time history of flapping wing kinematics. Two-dimensional contours show spanwise vorticial field at the section of $70 \%$ wing length for the wing root. Iso-voricity magnitude surface is employed to visualize threedimensional flow feature around the wing.

\section{Isotropic wing}

In order to investigate the impact of flapping isotropic wing on the aerodynamic force generation, an isotropic Zimmerman elliptic wing prescribed with pure flapping motion in a still air at $R e=1489$ and $k=0.56$ is simulated. The computational result is obtained using the serial version NS solver with thin plate spline interpolation remeshing 
method $^{18}$. The motion is excited at the rigid square at the leading edge of the wing with a sinusoidal motion; see Eq. (8). In this study, two effective stiffnesses are considered: $1.4 \times 10^{3}$ and $35.5 \times 10^{3}$. The density ratio and thickness ratio of structure is 2.7 and $2.0 \times 10^{-2}$, respectively.

All structured grid around the Zimmerman wing of aspect ratio 7.65 is used and the number of points in the converged grid configuration is 0.7 million approximately ${ }^{18}$. The basic computational setup is similar to one presented in Figure 11. For the outlet boundary condition, the pressure is set to zero. A no-slip condition on the flow velocity is assigned to the wing surface. The nodes on the symmetry plane, the purple colored plane in, are set to zero displacements.

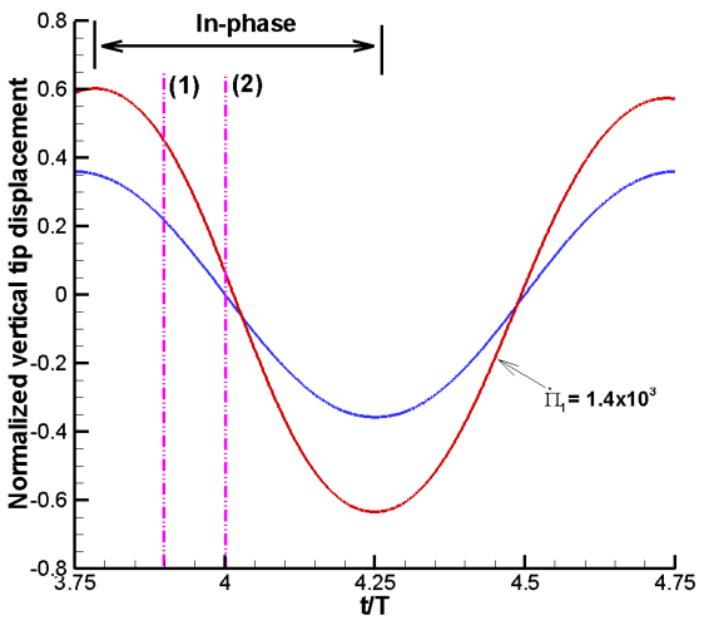

(1) Normalized vertical tip displacement

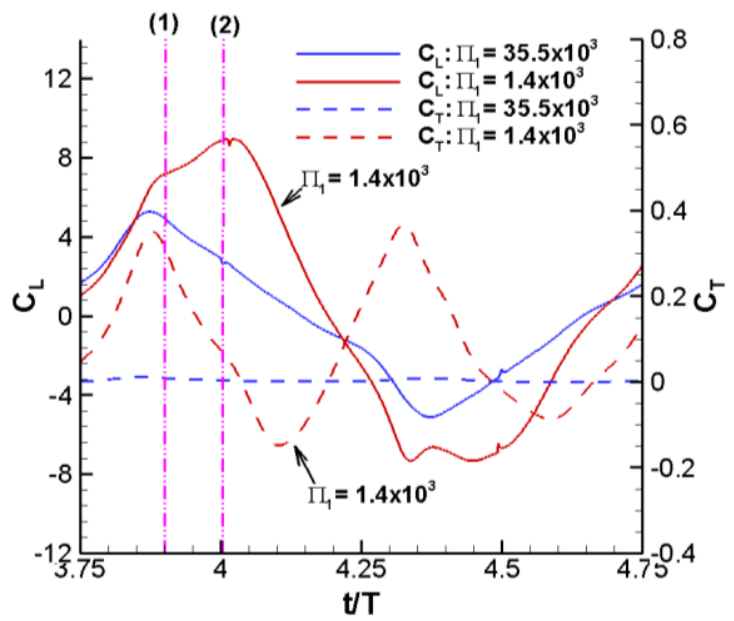

(2) Lift and drag coefficient

Figure 18 Structural and aerodynamic response of isotropic flapping wing. Tip displacement is normalized by the mean chord length. Blue and red lines correspond to effective stiffness of $1.4 \times 10^{3}$ and $35.5 \times 10^{3}$, respectively.

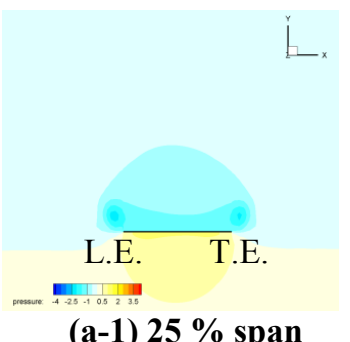

(a-1) $25 \%$ span

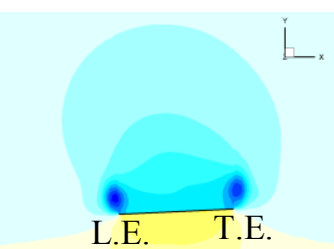

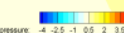

(b-1) $25 \%$ span

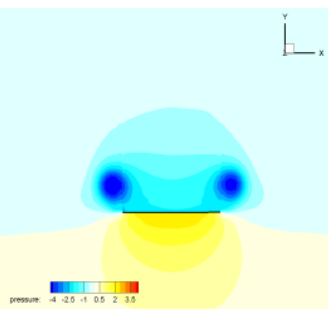

(a-2) $50 \%$ span

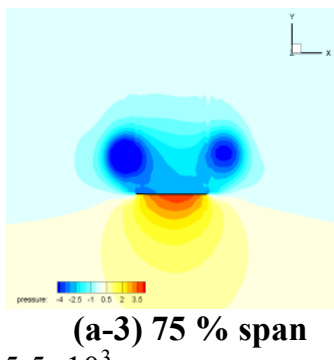

(a) $\Pi_{1}=35.5 \times 10^{3}$
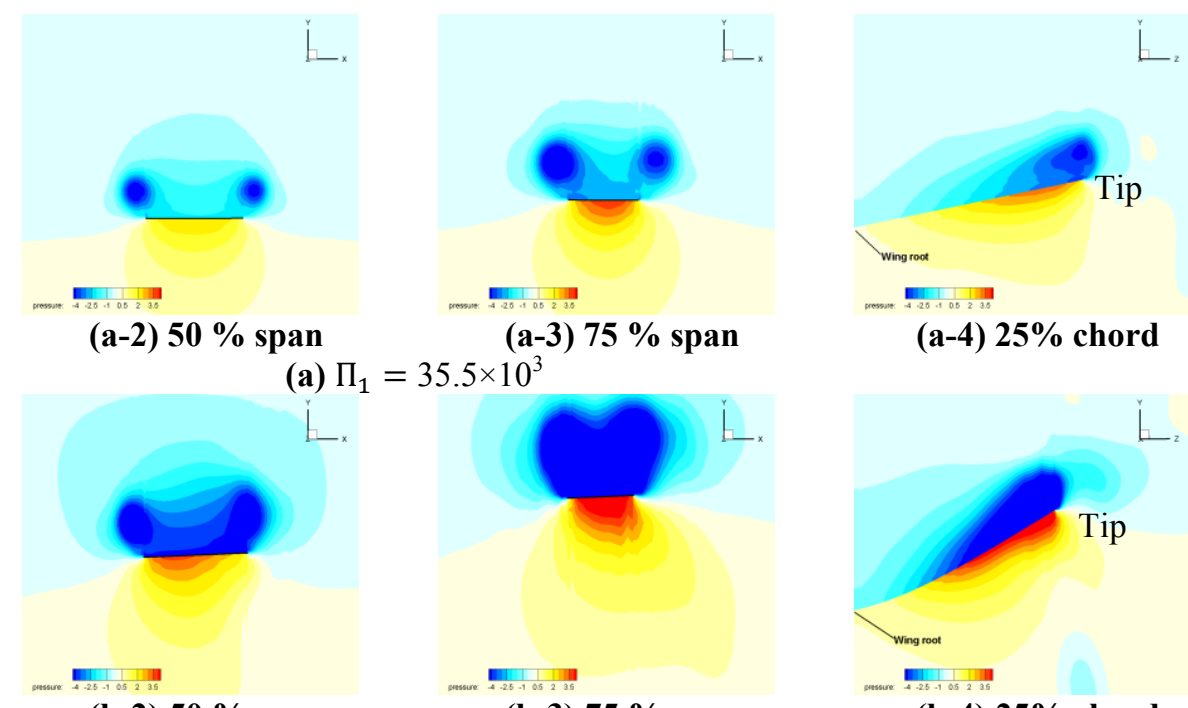

(b) $\Pi_{1}=1.4 \times 10^{3}$

(b-2) $50 \%$ span

(b-3) $75 \%$ span

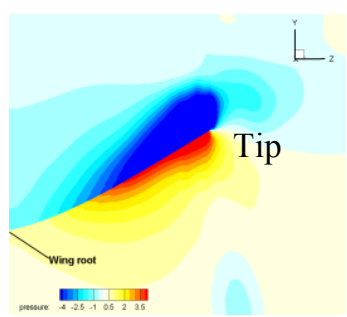

(b-4) $25 \%$ chord

Figure 19 Comparison of pressure distributions around flexible wings at maximum thrust generation during the donwstroke. L.E. and T.E. indicate leading and trailing edge of the wing.

Figure 18 presents the time histories of vertical tip displacement and aerodynamic coefficients of two flapping isotropic wing models over one flapping cycle. It is observed that the amplitude of instantaneous vertical tip displacement in the case of $\Pi_{1}=1.4 \times 10^{3}$ increases, while the period of tip motion remains the same. Therefore, the vertical tip velocity increases due to the wing flexibility. Coordination between the prescribed wing motion and the 
resultant tip movement shows in-phase motion during most of the stroke (see Figure 18 (a)). Previously Aono et al. ${ }^{4}$. showed for plunging spanwise flexible wing that coordinated motion between the deformed wing and the rigidly excited root is the underlying key mechanism for the flexibility enhanced aerodynamic force generation. Looking at time histories of lift and drag coefficients, clearly, the case of $\Pi_{1}=1.4 \times 10^{3}$ produces thrust and larger lift than that of $\Pi_{1}=35.5 \times 10^{3}$. (see Figure 18 (b)). This difference in the force generation is elicit in Figure 19 that shows the pressure distributions at the three selected chordwise sections $(25 \%, 50 \%, 75 \%)$ and one spanwise section $(25 \%)$ when the wing generates high thrust. It is found that for the case of $\Pi_{1}=1.4 \times 10^{3}$ flexibility induces positive pitching angle (see Figure 19 (b-1, 2, 3)). This pitching angle changes the direction of resulting aerodynamic force contributed by pressure favorable for the thrust generation. It should be mentioned that there is almost no camber of the wing due to wing flexibility. This is consistent with the previous findings by Shyy et al. ${ }^{2}$ that the chordwise flexibility elevates thrust generation by increased projected area of the deformed wing perpendicular to the freestream. Figure 20 illustrates the pressure distributions at the same selected chordwise and spanwise sections when the wing generates the maximal lift. Due to the larger bending of the wing tip (see Figure 18 (a)) the wing tip velocity increases, producing stronger vortex generation (i.e. leading edge, trailing edge, and tip vortices) and hence lower pressure region on the suction side of the wing surface. This results in larger resulting aerodynamic force generation. It can be seen that the flexibility-induced pitching angle is almost zero (see Figure 20 (b-1, 2, 3)). Therefore, most of the resultant aerodynamic force is projected to the direction perpendicular to the chord, the lift.

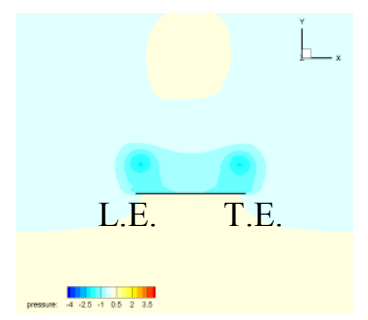

(a-1) $25 \%$ span

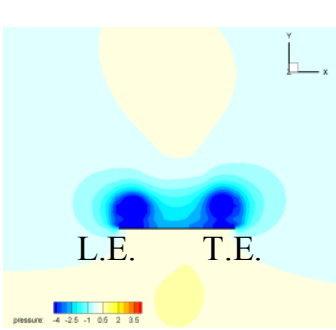

(b-1) $25 \%$ span

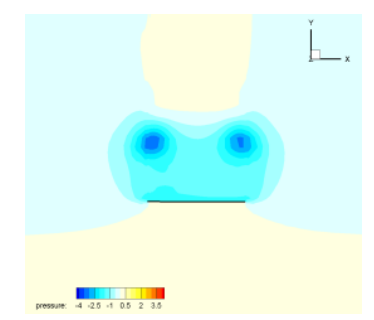

(a-2) $50 \%$ span

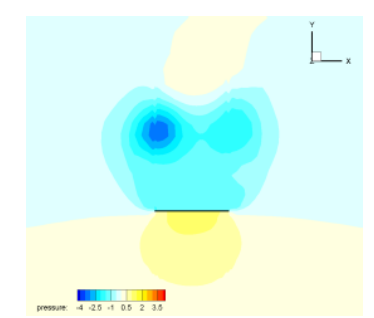

(a-3) $75 \%$ span

(a) $\Pi_{1}=35.5 \times 10^{3}$

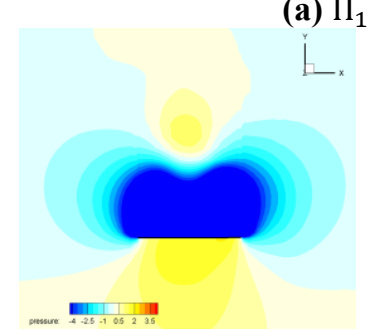

(b-2) 50 \% span

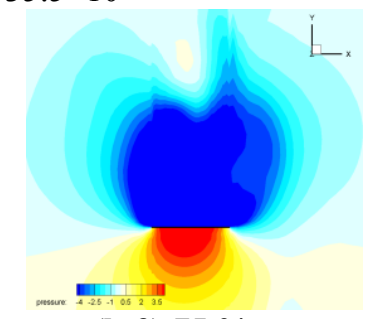

(b-3) $75 \%$ span

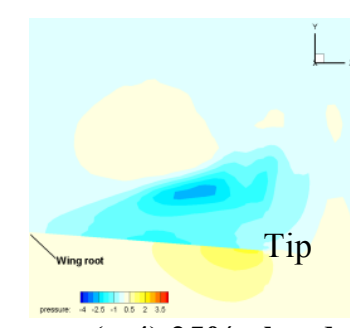

(a-4) $25 \%$ chord

(b) $\Pi_{1}=1.4 \times 10^{3}$

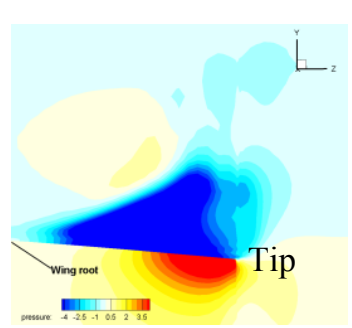

(b-4) $25 \%$ chord

Figure 20 Comparison of pressure distributions around flexible wings at maximum lift generation during the downstroke. L.E. and T.E. indicate leading and trailing edge of the wing.

\section{Summary and Conclusion}

Refining the computational framework reported in Ref 10, in present effort, we have incorporated an unstructured grid Navier-Stokes solver, significantly improved remeshing techniques, and anisotropic structural modeling capabilities. A remeshing method based on radial basis function interpolation shows to capable of handling large deformations, retaining initial grid qualities, and being computationally efficient. Flexible flapping wings operating in both vacuum and air are analyzed to shed light on inertial and aerodynamic loading-induced shape deformation. For the study of a flapping anisotropic wing in vacuum, a composite structural model is implemented based the classical laminated plate theories ${ }^{39}$ to account the anisotropic nature of wing structure. Results show good agreement with the experimental data ${ }^{12}$ in terms of instantaneous vertical tip deflection and location. Furthermore, the deflection and movement of the leading edge batten are seen to be the main driver of the overall wing motion, whereas the influence of deformations in the membrane and the chordwise batten is significant at the end and start of the strokes. Therefore, highly three-dimensional wing deformations are observed when the wing accelerates or decelerates in the absent of aerodynamic forces. 
For the flapping Zimmerman rigid wing in still air at $R e=1.5 \times 10^{3}$ and $k=0.56$ the aerodynamic force generation is coupled with the vortex generation due to the wing motion. For the flapping isotropic Zimmerman wing in still air, impacts of wing flexibility on the aerodynamic force generation is investigated. Two effective stiffnesses are considered: $1.4 \times 10^{3}$ and $35.5 \times 10^{3}$. The density ratio and thickness ratio of structure are 2.7 and $2.0 \times 10^{-2}$, respectively. In Refs 2 and 4, the implications of chordwise ${ }^{2}$ and spanwise ${ }^{4}$ flexibility on aerodynamics were investigated. Overall, the thrust generation consists of contributions due to both leading edge suction and the pressure projection of the chordwise deformed rear foil. Even though the effective angle of attack and the net aerodynamic force are reduced due to chordwise shape deformation, both mean and instantaneous thrust can be enhanced due to the increase of projected area normal to the flight trajectory. For the spanwise flexible case, correlations of the motion from the root to the tip play a role. Within a suitably selected range of spanwise flexibility, the effective angle of attack and thrust forces of a plunging wing are enhanced due to the wing deformations. These findings are in broad agreement with those observed in the present study: For an isotropic wing two consistent mechanisms which can enhance thrust and lift are identified, namely

1) higher wing velocity due to larger bending motion can enhance the aerodynamic force,

2) flexibility-induced pitching angle can elevate thrust.

\section{Acknowledgments}

The work supported here has been supported in part by the Air Force Office of Scientific Research's Multidisciplinary University Research Initiative (MURI) grant and by the Michigan/AFRL (Air Force Research Laboratory) Collaborative Center in Aeronautical Sciences. We thank Mr. Trizila and Dr. Wright for the fruitful discussions provided.

\section{References}

1. Shyy, W., Lian, Y., Tang, J., Viieru, D., and Liu, H., Aerodynamics of low Reynolds number flyers New York: Cambridge University Press, 2008.

2. Shyy, W., Aono, H., Chimakurthi, S.K., Trizila, P., Kang, C., Cesnik, C.E.S., and Liu, H., "Recent Progress in Flapping Wing Aerodynamics and Aeroelasticity," Progress in Aerospace Sciences, In Press, 2010, doi:10.1016/j.paerosci.2010.01.001

3. Shyy, W., Lian, Y., Tang, J., Liu, H., Trizila, P., Stanford, B., Bernal, L., Cesnik, C.E.S., Friedmann, P., and Ifju, P., "Computational Aerodynamics of Low Reynolds Number Plunging, Pitching and Flexible Wings For MAV Applications," Acta Mechanica Sinica, Vol. 24, pp.351-373, 2008.

4. Aono, H., Chimakurthi, S.K., Cesnik, C.E.S., Liu, H., and Shyy, W., "Computational Modeling of Spanwise Effects on Flapping Wing Aerodynamics," AIAA 2009-1270.

5. Ishihara, D., Yamashita, Y., Horie, T., Yoshida, S., and Niho, T., "Passive Maintenance of High Angle of Attack and its Lift Generation during Flapping Translation in Crane Fly Wing," Journal of Experimental Biology, Vol. 212, pp. 3882-3891, 2009.

6. Gopalakrishnan, P., Tafti, D.K, "Effect of Wing Flexibility on Lift and Thrust Production in Flapping Flight," AIAA Journal, Vol. 48, No. 5, 2010.

7. Combes, S.A., Daniel, T.L., "Flexural Stiffness in Insect Wings I. Scaling and the Influence of Wing Venation," Journal of Experimental Biology, Vol. 206, pp. 2979-2987, 2003.

8. Combes, S.A., Daniel, T.L., "Flexural Stiffness in Insect Wings II. Spatial Distribution and Dynamic Wing Bending," Journal of Experimental Biology, Vol. 206, pp. 2989-2997, 2003.

9. Heathcote, S., Wang, Z., and Gursul, I., "Effect of Spanwise Flexibility on Flapping Wing Propulsion," Journal of Fluid \& Structures, Vol. 24, pp. 183-199, 2008.

10. Chimakurthi, S.K., Tang, J., Palacios, R., Cesnik, C.E.S., Shyy W., "Computational Aeroelasticity Framework for Analyzing Flapping Wing Micro Air Vehicles," AIAA Journal, Vol. 47, Nr. 8, pp. 1865-1878, 2009.

11. Zhu, Q., "Numerical Simulation of a Flapping Foil with Chordwise or Spanwise Flexibility," AIAA Journal, Vol. 45, No. 10, pp. 2448-2457, 2007.

12. Pin, W., Ifju P., Stanford, B., Sällström, E., Ukeiley, L., Love, R., and Lind, R., "A Multidisciplinary Experimental Study of Flapping Wing Aeroelasticity in Thrust Production," AIAA -2009-2413.

13. Anderson, J.D., Fundamentals of Aerodynamics, Second Edition, 1991, Singapore.

14. Ishihara, D., Yamashita, Y., Horie, T., Yoshida, S., and Niho, T., "A Two-dimensional Computational Study on the Fluid-Structure Interaction Cause of Wing Pitch Changes in Dipteran Flapping Wing," The Journal of Experimental Biology, Vol. 212, pp. 3882-3891, 2009. 
15. Lentink, D., and Dickinson, M.H., "Biofluiddynamic Scaling of Flapping, Spinning and Translating Fins and Wings," The Journal of Experimental Biology, Vol. 212, pp. 2691-2704, 2009.

16. Tang, J., Chimakurthi, S., Palacios, R., Cesnik, C.E.S., and Shyy, W., "Computational Fluid-Structure Interaction of a Deformable Flapping Wing for Micro Air Vehicle Applications," AIAA 2008-615.

17. Chimakurthi, S.K., Stanford, B.K., Cesnik, C.E.S., and Shyy, W., "Flapping Wing CFD/CSD Aeroelastic Formulation Based on a Co-rotational Shell Finite Element," AIAA 2009-2412.

18. Aono, H., Chimakurthi, S.K., Wu, P., Sällström, E., Stanford, B.K., Cesnik, C.E.S., Ifju, P., Ukeiley, L, and Shyy, W., "A Computational and Experimental Study of Flexible Flapping Wing Aerodynamics," AIAA 2010-554.

19. Gordnier, R.E., Attar P.J., Chimakurthi, S.K., and Cesnik, C.E.S., "Implicit LES Simulations of a Flexible Flapping Wing," AIAA 2010-2060.

20. McClung, A., Stanford, B., and Beran, P, "High-Fidelity Models for the Fluid-Structure Interaction of a Flexible Heaving Airfoil," AIAA 2010-2959.

21. Stanford, B., Kurdi, M., Beran, P., and McClung, A., "Shape-Structure, and Kinematic Parameterization of a Power-Optimal Hovering Wing,” AIAA 2010-2963.

22. K. Stein, T. Tezduyar and R. Benney, "Mesh Moving Techniques for Fluid-Structure Interactions with Large Displacements," Journal of Applied Mechanics, Vol. 70, pp. 58-63, 2003.

23. Smith, R.W., and Wright, J.A., "A Classical Elasticity-Based Mesh Update Method for Moving and Deforming Meshes," AIAA 2010-164.

24. de Boer, A., van der Schoot, M.S., and Bijl, H., "Mesh Deformation Based on Radial Basis Function Interpolation," Computers and Structures, Vol. 85, pp. 784-795, 2007.

25. Rendall, T.C.S., and Allen, C.B., "Unified Fluid-Structure Interpolation and Mesh Motion using Radial Basis Functions," International Journal for Numerical Methods in Engineering, Vol. 74, pp. 1519-1559, 2008.

26. Rendall, T.C.S., and Allen, C.B., "Efficient Mesh Motion Using Radial Basis Functions with Data Reduction Algorithms," Journal of Computational Physics, Vol. 228, pp. 6231-6249, 2009.

27. Rendall, T.C.S., and Allen, C.B., "Reduced Surface Point Selection Options for Efficient Mesh Deformation using Radial Basis Functions," Journal of Computational Physics, Vol. 229, pp. 2810-2820, 2010.

28. Wright, J.A., and Smith, R.W., "An Edge-Based Method for the Incompressible Navier-Stokes Equations on Polygonal Meshes,” Journal of Computational Physics, Vol. 169, pp. 24-43, 2001.

29. Kamakoti, R., and Shyy, W., "Evaluation of Geometric Conservation Law using Pressure-Based Fluid Solver and Moving Grid Technique", International Journal of Numerical Methods for Heat \& Fluid Flow, Vol. 14, No. 7, p. 851-865, 2006.

30. Luke, E.A., and George, T., "Loci: a rule-based framework for parallel multi-disciplinary simulation synthesis," Journal of Functional Programming, Vol. 15, Nr. 3, pp.477-502, 2005.

31. Shyy, W., "A Study of Finite Difference Approximations to Steady-State, Convection-Dominated Flow Problems", Journal of Computational Physics, Vol. 57, No. 3, 1985, pp. 415-438.

32. Shyy, W., Computational Modeling for Fluid Flow and Interfacial Transport, Elsevier, Amsterdam, 1994.

33. Blazek, J., Computational Fluid Dynamics: Principles and Applications, Elsevier, Amsterdam, 2001.

34. Thomas, P.D., and Lombard, K., "The Geometric Conservation Law - A Link between Finite-Difference and Finite-Volume Methods of Flow Computation on Moving Grids", AIAA paper 1978-1208, July 1978.

35. Balay, S., Buschelman, K., Gropp, W.D., Kaushik, D., Knepley, M.G., McInnes, L.C., Smith, B.F., and Zhang, H, "PETSc Web page", http://www.mcs.anl.gov/petsc, 2009.

36. Kamakoti, R., and Shyy, W., "Evaluation of Geometric Conservation Law using Pressure-Based Fluid Solver and Moving Grid Technique", International Journal of Numerical Methods for Heat \& Fluid Flow, Vol. 14, No. 7, p. 851-865.

37. Smith, R.W., and Wright, J.A., "An Implicit Edge-Based ALE Method for the Incompressible Navier-Stokes Equations," Int. J. Numer. Meth. Fluids, Vol 43, pp.253-279, 2003.

38. Beckert, A., and Wendland, H., "Multivariate Interpolation for Fluid-Structure-Interaction Problems using Radial Basis Functions," Aerospace Science and Technology, Vol. 5, Nr. 2, pp. 2-8, 2000.

39. Berthelot, J.-M., Composite Materials, Springer 1997.

40. Gogulapati, A., Friedmann, P. P., Khen, E., and Shyy W., "Approximate Aeroelastic Modeling of Flapping Wing Comparison with CFD and Experimental Data," AIAA 2010-2707.

41. Liu, H., and Aono, H., "Size effects on insect hovering aerodynamics: an integrated computational study," Bioinspiration and Biomimetics, Vol.4, No. 1, pp.1-13, 2009. 